\title{
Local real analysis in locally homogeneous spaces
}

\author{
Marco Bramanti and Maochun Zhu
}

May 29, 2018

\begin{abstract}
We introduce the concept of locally homogeneous space, and prove in this context $L^{p}$ and $C^{\alpha}$ estimates for singular and fractional integrals, as well as $L^{p}$ estimates on the commutator of a singular or fractional integral with a $B M O$ or $V M O$ function. These results are motivated by local a-priori estimates for subelliptic equations.
\end{abstract}

\section{Introduction}

\section{Motivation}

The theory of singular integrals has been usefully applied to local a priori estimates for PDEs in several contexts of increasing generality, in the last decades. The abstract framework of spaces of homogeneous type, introduced by CoifmanWeiss in [19, has proved to be a suitable framework in many cases, so far: we have a set (which in concrete applications is usually a bounded domain of $\mathbb{R}^{n}$ ), a distance or a quasidistance adapted to the differential operator (the Euclidean distance for classical elliptic equations, parabolic distance for parabolic equations, Carnot-Carathéodory distance -or some variation of it- for operators built on Hörmander's vector fields -see [26]-, and so on), and a measure (usually the Lebesgue measure) which is doubling with respect to the metric balls. In these situations the quasidistance $\rho$ is usually defined in some $\Omega_{0}$ which is either the whole $\mathbb{R}^{n}$ or some domain which is larger than the bounded domain $\Omega$ where we want to prove our estimates. Since the balls $B(x, r)$ are, by definitions, subsets of $\Omega_{0}$, that is

$$
B(x, r)=\left\{y \in \Omega_{0}: \rho(x, y)<r\right\},
$$

if we want to apply the theory of spaces of homogeneous type to the set $\Omega$, the doubling condition we have to check is

$$
\mu(B(x, 2 r) \cap \Omega) \leq c \mu(B(x, r) \cap \Omega) \text { for any } x \in \Omega, r>0 .
$$

On the other hand, the doubling condition that reasonable $\rho$ and $\mu$ usually satisfy is

$$
\mu(B(x, 2 r)) \leq c \mu(B(x, r)) \text { for any } x \in \Omega, 0<r<r_{0}
$$


for some small $r_{0}$. Passing from (2) to (1) requires some "smoothness" property of $\partial \Omega$, a property which, however, is not a natural requirement for our original local problem, but more a technical complication due to the fact that, in order to apply the theory of spaces of homogeneous type, we are regarding the set $\Omega$, which in our problem is a subset of a larger universe, as the universe itself. If $\mu$ is the Lebesgue measure and $\rho$ is the Euclidean distance, in order to fulfil (1) it is enough to require $\partial \Omega$ Lipschitz; if $\rho$ is the Carnot-Carathéodory distance induced by a set of Hörmander's vector fields $X_{1}, X_{2}, \ldots, X_{q}$ which is naturally attached to the study of the operator

$$
\sum_{i=1}^{q} X_{i}^{2}
$$

then (1) is satisfied for instance when $\Omega$ is itself a metric ball, as was first proved in [21] (see also [4, Lemma 4.2] for a more self-contained proof); this result basically relies on the fact that this quasidistance has the segment property, which essentially means that for any couple of points $x_{1}, x_{2}$ at distance $r$ and for any number $\delta<r$ there exists a point $x_{0}$ having distance $\delta$ from $x_{1}$ and $r-\delta$ from $x_{2}$. An analog result of regularity for the metric ball has been proved in [5] for the "parabolic Carnot-Carathéodory distance" attached to the operator

$$
\partial_{t}-\sum_{i=1}^{q} X_{i}^{2}
$$

This distance has no longer the segment property, but the simple way in which the time variable is involved allows to do explicit (but nontrivial!) computations and show that when $\Omega$ is a metric ball, (11) still holds.

If we now pass to consider Hörmander's operators of the kind

$$
\sum_{i=1}^{q} X_{i}^{2}+X_{0}
$$

(where the drift term $X_{0}$ is part of the set $X_{0}, X_{1}, \ldots, X_{q}$ which satisfies Hörmander's condition), the corresponding quasidistance $\rho$ is the one defined by Nagel-SteinWainger in 26; this quasidistance has been much less studied than the usual Carnot-Carathéodory distance (we can quote, in the context of nonsmooth Hörmander's vector fields, the two papers [6, 7]). Although a local doubling condition (2) holds, as proved in 26, this quasidistance does not satisfy the segment property and, as far as we know, a condition of kind (1) has never been proved for $\Omega$ a metric ball, or for any other special kind of bounded domain $\Omega$. Therefore the existing results do not allow to apply the theory of spaces of homogeneous type to the space $(\Omega, \rho, \mu)$ when $\Omega$ is some bounded domain of $\mathbb{R}^{n}$, $\rho$ is the Nagel-Stein-Wainger distance attached to the set of Hörmander's vector fields $X_{1}, X_{2}, \ldots, X_{0}$ (with $X_{0}$ of "weight" two), and $\mu$ the Lebesgue measure. This problem has been sometimes overlooked, apparently; for instance, in the famous paper [27], $L^{p}$ estimates are proved for operators (3), and stated for 
operators (4), without any reference to the mere existence of the problem of assuring the validity of condition (1). On the other hand, as already suggested, one feels that if our final goal is that of proving local estimates, no kind of "smoothness" of the domain $\Omega$ with respect to the quasidistance should be a crucial requirement; in other words, it is reasonable that this difficulty could be bypassed. The basic scope of the present paper is to build up a local theory of singular integrals which does not require checking condition (1), when (2) is known.

Another problem of a-priori estimates for PDEs in which proving that (2) implies (1) for some domain $\Omega$ appears troublesome has been studied in [10. In that paper the Authors consider a class of Kolmogorov-Fokker-Planck operators on $\mathbb{R}^{n} \times[-1,1]$, for which the natural quasidistance is a function $\rho$ (not equivalent to the Carnot-Carathéodory distance induced by a system of Hörmander's vector fields), which satisfies the quasi-triangle inequality on any compact set and satisfies a local doubling condition (2) for any bounded $\Omega$; again, however, one has no idea of how to prove (1) for some particular bounded $\Omega$. In that case, the Authors overcame the problem by applying an ad-hoc theory of singular integrals in nondoubling spaces, developed in [2]. The resort to theories of singular integrals in nondoubling contexts, as have been developed in the last decade by Tolsa, Nazarov-Treil-Volberg, and other authors (see for instance the book 29] and references therein), is actually an alternative possibility in order to bypass (11). However, and here another motivation of the present paper comes in, when proving a-priori $L^{p}$ estimates for PDEs with $V M O$ coefficients (a line of research which started with the papers by Chiarenza-Frasca-Longo [14, [15] and developed in several directions in the last 20 years), one needs a series of results about commutators of singular and fractional integrals with $B M O$ functions, which do not have a natural counterpart in the nondoubling context; more precisely, results of this kind have been actually proved by Tolsa [28], in the context of $\left(\mathbb{R}^{n}, d, d \mu\right)$, where $\mu$ is a very general Radon measure, but $d$ is the Euclidean distance. Since the extension of these deep results to the case of a general quasidistance $d$ appears far from being obvious, it seems easier and more natural for the problem under exam to establish these commutator theorems in the framework of a theory of singular integrals in a locally doubling context (instead than in a nondoubling one). More generally, we think that the idea of proving a local version of some basic results about singular integrals is a very natural one, and we feel that these results can be of some interest also for other applications.

\section{Main results}

In this paper we will prove, in the context of locally homogeneous spaces (which will be defined in the next section), results of continuity, in $L^{p}$ and $C^{\alpha}$ spaces, for singular and fractional integrals, as well as $L^{p}$ estimates for the commutator of a singular or fractional integral with the multiplication with a $B M O$ function. Also, we will state these commutator theorems in a form suitable to prove the smallness of the $L^{p}$ norm of the commutator on a small ball, whenever the $B M O$ 
function actually belongs to $V M O$. This localized version of the commutator theorem for a $V M O$ function, in the original Euclidean case (exploited in [14, [15]) relies on the possibility of approximating a $V M O$ function by a uniformly continuous function in $B M O$ norm, and on the possibility of extending to the whole space a uniformly continuous function defined on a ball, preserving the continuity modulus. Here we manage to establish directly the localized version of the commutator theorems for a $V M O$ function, without the necessity of proving the aforementioned approximation and extension results. Therefore, under this respect, our approach conceptually simplifies also the Euclidean case. We will also deal, very briefly, with a local version of the maximal function and its $L^{p}$ continuity, another tool which is useful in the alluded applications.

Our main results are: Theorems 21 and 24 in Section [5. Theorems 29, 30. 31 in Section 7, Theorem 35] in Section 8. We also think that the basic theory developed in Sections 2 23, particularly Theorems 6 and 15, could be useful to prove further results in the same spirit.

All the results proved in this paper will be used in the proof of $L^{p}$ and $C^{\alpha}$ estimates for nonvariational operators structured on Hörmander's operators of type (4), that is for operators of the form

$$
\sum_{i . j=1}^{q} a_{i j}(x) X_{i} X_{j}+a_{0}(x) X_{0}
$$

where $X_{0}, X_{1}, \ldots, X_{q}$ are a system of smooth Hörmander's vector fields in a bounded domain of $\mathbb{R}^{n}(n>q+1),\left\{a_{i j}\right\}$ is a uniformly positive matrix with bounded entries, $a_{0}$ is bounded and bounded away from zero and all the coefficients $a_{i j}, a_{0}$ belong to the suitable function space $V M O$ or $C^{\alpha}$ (respectively, to prove $L^{p}$ and $C^{\alpha}$ estimates on $X_{i} X_{j} u$ ). These estimates are proved in a separate paper [1], and generalize the results proved in 3 and [5] when the drift $X_{0}$ is lacking.

\section{Strategy}

The basic idea, in order to bypass the necessity of checking condition (11) instead of (2), is to adapt the abstract construction of dyadic cubes in spaces of homogeneous type carried out by Christ in [16]. In that paper, the Author shows how in any space of homogeneous type one can construct, for any $k \in \mathbb{Z}$, a family of "dyadic cubes" of diameter comparable to $\delta^{k}$ (with $\delta$ a small fixed number). Actually these "cubes" are open sets, defined by an abstract construction, which nevertheless share with the classical dyadic cubes all the basic properties. The relevant fact for us is that each of these cubes $Q$ is, in turn, a space of homogeneous type, that is in [16] the Author proves that (11) actually holds for $\Omega=Q$. Here we adapt the previous construction in a local setting. Given our space $\Omega$, which is seen as the union of an increasing sequence of bounded subsets $\Omega_{n}$, we construct for each $n$ and each scale $k=1,2,3 \ldots$, a family of (small) dyadic cubes essentially covering $\Omega_{n}$ and contained in $\Omega_{n+1}$; each of these cubes can still be proved to be a space of homogeneous type; moreover, the same is true 
for any finite union of dyadic cubes of the same scale $k$. The idea is then to apply known results for spaces of homogeneous type to suitable unions of dyadic cubes which cover a fixed small ball, and derive the corresponding result on the ball. Since dyadic cubes are abstract objects, which in concrete applications of the theory cannot be explicitly exhibited, our job is to use dyadic cubes just as a tool, but to state and prove all our results in the language of balls, to make them easily applicable.

To make more transparent the strategy of our construction, let us point out what follows.

We will show that for any $n$ we can cover $\Omega_{n}$ with a finite union of balls of any prescribed small size, and for each of these balls we can construct a space of homogeneous type $F$ which is contained in $\Omega_{n+1}$, "almost contains" this ball $B$, and is comparable to $B$, both in measure and in diameter. This "almost inclusion" is made precise in two ways:

1) $F \supset B \backslash E$ where $E$ is a zero measure set; this inclusion is enough to handle $L^{p}$ estimates or more generally estimates which involve integral norms;

2 ) the closure of $F$ contains $B$; this inclusion is enough to handle $C^{\alpha}$ estimates, or more generally estimates which involve moduli of uniform continuity of the functions.

The idea of exploiting Christ's construction of dyadic cubes to prove results in a locally doubling context has been already used by Carbonaro, Mauceri, Meda in [12]; their context, however, is different from ours: in that paper the Authors consider a situation where the measure of balls grows fast at infinity, so that the doubling condition holds for balls of radius $r \leq r_{0}$, for any fixed $r_{0}$; on the other hand, these Authors have not our problem of keeping far from the boundary of a bounded domain, to avoid intersections. Moreover, they use dyadic cubes to adapt the proofs which hold in the doubling case, while our strategy is not to adapt the existing proofs but to apply the existing results which hold in the doubling case.

The construction of a suitable family of spaces of homogeneous type is not the only problem to overcome in our situation. The possible overlapping of the balls $B(x, r)$ with the boundary of the considered domain creates problems under at least other two regards. The first is the validity of a suitable cancellation property for the kernel of singular integral operator: if we know that a singular kernel has a bounded integral over small spherical shells, this does not imply the persistence of this property when we integrate over the intersection of the spherical shell with some fixed domain. This problem will be solved using suitable cutoff functions. The second problem is to suitably define and handle $B M O$ and $V M O$ spaces, avoiding to take the average of a function over the intersection of $B(x, r)$ with a fixed domain. To this aim we will introduce a $B M O_{l o c}$ space adapted to a couple of domains $\Omega_{n} \subset \Omega_{n+1}$, which in our context is a natural notion, and we will show which relation this space has with the standard $B M O$. 


\section{Plan of the paper}

In Section 2 we state precisely our definition of locally homogeneous spaces and draw some first consequences of the definition, in terms of topology and measure. Section 3 contains the construction of dyadic cubes and the proof of their relevant properties which will allow to apply the theory of spaces of homogeneous type. In Section 4 we build, in a fairly standard way, Hölder continuous cutoff functions, another tool which will be useful in the following. In Section 5 we prove our local $L^{p}$ and $C^{\alpha}$ continuity results for singular and fractional integrals. In Section 6 we introduce $B M O$ and $V M O$ spaces, both in the standard and in a local version, and study the relation between the two concepts. In Section 7 we prove local $L^{p}$ estimates on the commutator of a singular or fractional integral with a $B M O$ or $V M O$ function. In Section 8 we deal with the local maximal operator and its $L^{p}$ continuity. Finally, in Section 9 we extend the results of Sections 5 to 8 to the more general situation where the local quasidistance is quasisymmetric but not symmetric. An Appendix collects all the known results about spaces of homogeneous type which we need throughout the paper.

Acknowledgements. This research was mainly carried out while Maochun Zhu was visiting the Department of Mathematics of Politecnico di Milano, which we wish to thank for the hospitality. The project was supported by the National Natural Science Foundation of China (Grant No. 10871157), Specialized Research Fund for the Doctoral Program of Higher Education (No. 200806990032).

\section{The abstract framework of locally homoge- neous spaces}

We are going to state the assumptions which will define the notion of locally homogeneous space. (For comparison, the standard definition of space of homogeneous type is recalled in the Appendix, Section 10).

(H1) Let $\Omega$ be a set, endowed with a function $\rho: \Omega \times \Omega \rightarrow[0, \infty)$ such that for any $x, y \in \Omega$ :

(a) $\rho(x, y)=0 \Leftrightarrow x=y$;

(b) $\rho(x, y)=\rho(y, x)$.

For any $x \in \Omega, r>0$, let us define the ball

$$
B(x, r)=\{y \in \Omega: \rho(x, y)<r\} .
$$

These balls can be used to define a topology in $\Omega$, saying that $A \subset \Omega$ is open if for any $x \in A$ there exists $r>0$ such that $B(x, r) \subset A$. Also, we will say that $E \subset \Omega$ is bounded if $E$ is contained in some ball.

Let us assume that:

(H2) (a) the balls are open with respect to this topology;

(H2) (b) for any $x \in \Omega$ and $r>0$ the closure of $B(x, r)$ is contained in $\{y \in \Omega: \rho(x, y) \leq r\}$. 
We will prove in a moment that the validity of conditions (H2) (a) and (b) is equivalent to the following:

(H2') $\rho(x, y)$ is a continuous function of $x$ for any fixed $y \in \Omega$.

(H3) Let $\mu$ be a positive regular Borel measure in $\Omega$.

(H4) Assume there exists an increasing sequence $\left\{\Omega_{n}\right\}_{n=1}^{\infty}$ of bounded measurable subsets of $\Omega$, such that:

$$
\bigcup_{n=1}^{\infty} \Omega_{n}=\Omega
$$

and such for, any $n=1,2,3, \ldots$ :

(i) the closure of $\Omega_{n}$ in $\Omega$ is compact;

(ii) there exists $\varepsilon_{n}>0$ such that

$$
\left\{x \in \Omega: \rho(x, y)<2 \varepsilon_{n} \text { for some } y \in \Omega_{n}\right\} \subset \Omega_{n+1} ;
$$

(H5) there exists $B_{n} \geq 1$ such that for any $x, y, z \in \Omega_{n}$

$$
\rho(x, y) \leq B_{n}(\rho(x, z)+\rho(z, y)) ;
$$

(H6) there exists $C_{n}>1$ such that for any $x \in \Omega_{n}, 0<r \leq \varepsilon_{n}$ we have

$$
0<\mu(B(x, 2 r)) \leq C_{n} \mu(B(x, r))<\infty .
$$

(Note that for $x \in \Omega_{n}$ and $r \leq \varepsilon_{n}$ we also have $\left.B(x, 2 r) \subset \Omega_{n+1}\right)$.

Definition 1 We will say that $\left(\Omega,\left\{\Omega_{n}\right\}_{n=1}^{\infty}, \rho, \mu\right)$ is a locally homogeneous space if assumptions (H1) to (H6) hold.

Dependence on the constants. The numbers $\varepsilon_{n}, B_{n}, C_{n}$ will be called "the constants of $\Omega_{n}$ ". It is not restrictive to assume that $B_{n}, C_{n}$ are nondecreasing sequences, and $\varepsilon_{n}$ is a nonincreasing sequence. Throughout the paper our estimates, for a fixed $\Omega_{n}$, will often depend not only on the constants of $\Omega_{n}$, but also (possibly) on the constants of $\Omega_{n+1}, \Omega_{n+2}, \Omega_{n+3}$. We will briefly say that "a constant depends on $n$ " to mean this type of dependence.

In the language of [19, $\rho$ is a quasidistance in each set $\Omega_{n}$; we can also say that it is a local quasidistance in $\Omega$. We stress that the two conditions appearing in (H2) are logically independent each from the other, and they do not follow from (7), even when $\rho$ is a quasidistance in $\Omega$, that is when $B_{n}=B>1$ for any $n$. If, however, $\rho$ is a distance in $\Omega$, that is $B_{n}=1$ for any $n$, then (H2) is automatically fulfilled.

The continuity of $\rho$ also implies that (7) still holds for $x, y, z \in \bar{\Omega}_{n}$. We will sometimes exploit this fact.

Also, note that $\mu\left(\Omega_{n}\right)<\infty$ for every $n$, since $\bar{\Omega}_{n}$ is compact. (This follows by the regularity of $\mu$, or also from the finiteness of the measure of balls, see (H6), since $\bar{\Omega}_{n}$ can be covered by a finite number of small balls). 
We also point out that assuming $\mu$ regular (see (H3)) is not really necessary, as will be explained after Proposition 18. however, since this assumption is harmless in the applications we are interested in, we prefer to keep it, in order to avoid the necessity of entering into annoying details.

Examples 2 (i) If $(\Omega, \rho, d \mu)$ is a bounded space of homogeneous type in the sense of Coifman-Weiss [19] (the definition will be recalled in the Appendix), the above conditions are fulfilled choosing $\Omega_{n} \equiv \Omega$ and $B_{n}=B \geq 1$ for any $n$.

(ii) In the applications to subelliptic equations that we have in mind, and will be dealt in [11], $\Omega$ is a bounded domain of $\mathbb{R}^{N}$ and $\Omega_{n}$ an increasing sequence of bounded domains, with $\Omega_{n} \Subset \Omega_{n+1} \Subset \Omega$ for any $n ; \rho$ is the Nagel-SteinWainger distance induced by a family $X_{0}, X_{1}, X_{2}, \ldots, X_{q}$ of Hörmander's vector fields, where $X_{0}$ has weight two, $\mu$ the Lebesgue measure in $\mathbb{R}^{N}$.

(iii) The same setting of (ii) fits the theory of nonsmooth Hörmander's vector fields, as dealt in [6], [7].

Note that in the situations (ii)-(iii) $\rho$ is actually a distance, which induces the Euclidean topology, and (H6) is a known result.

(iv) In the situation considered in [10], $\Omega=\mathbb{R}^{N} \times[-1,1], \Omega_{n}=B_{n} \times$ $[-1,1]$ where $B_{n}$ is the Euclidean ball of center 0 and radius $n$ in $\mathbb{R}^{N}, \rho(z, \zeta)=$ $\left\|\zeta^{-1} \circ z\right\|$ where $\circ$ is a Lie group operation related to the differential operator

$$
L=\sum_{i, j=1}^{p_{0}} a_{i j} \partial_{x_{i} x_{j}}^{2}+\sum_{i, j=1}^{N} b_{i j} x_{i} \partial_{x_{j}}-\partial_{t} \quad\left(\text { where } p_{0}<N\right)
$$

which is under study, while $\|\cdot\|$ is the homogeneous norm defined by the family of dilations related to another differential operator, which is the "principal part" of $L$. Therefore $\rho$ is neither the usual distance considered in Carnot groups, nor is (equivalent to) the Carnot-Carathéodory distance induced by the vector fields; $\rho$ satisfies (H5) and induces the Euclidean topology; from its analytical definition it is clear that $\rho$ is continuous, hence (H2) is fulfilled; also (H6) can be proved. More precisely, the function $\rho$ considered in [10] is not symmetric but satisfies a weaker condition: for any $n$ there exists $A_{n}>1$ such that

$$
\rho(x, y) \leq A_{n} \rho(y, x) \text { for any } x, y \in \Omega_{n} .
$$

This motivates a further extension of our theory, as we will explain in a moment.

Remark 3 (Extension to quasisymmetric functions) As anticipated in the above Example (iv), in view of some applications it is desirable to consider the more general setting in which $\rho$ is not assumed symmetric, but satisfies condition (9). However, developing the whole theory of Section 3 under this weaker assumption would make our computations considerably heavier. Instead, it is much easier to develop first the theory under the symmetry assumption, and then to show that our main results about singular and fractional integrals still hold if we replace the symmetry condition with (9). This extension will be discussed in Section 9. 
In the rest of this section we will make some remarks and prove some easy facts related to topology and measure in a locally homogeneous space.

Since, by (H2) (a), the balls are open, for each $x \in \Omega$ the balls $B(x, r)$ satisfy the axioms of complete system of neighborhoods of $x$; hence the topological space $\Omega$ is first countable, and continuity and closedness can be discussed by means of sequences of points. Let us prove the following fact, that we have claimed before.

Proposition 4 Conditions (H2) are equivalent to condition (H2').

Proof. Assume (H2), and let us prove the continuity of $x \longmapsto \rho(x, y)$. Fix $x \in \Omega$ and take a sequence $\left\{x_{k}\right\}$ converging to $x$. Let us show that $\rho\left(x_{k}, y\right) \rightarrow \rho(x, y)$ for any $y \in \Omega$. Let $r=\rho(x, y)$ and $\varepsilon>0$; since $x \in B(y, r+\varepsilon)$ and the balls are open, there exists $B(x, \delta) \subset B(y, r+\varepsilon)$. Then $x_{k} \in B(y, r+\varepsilon)$ definitively, since $x_{k} \rightarrow x$. This implies that $\rho\left(x_{k}, y\right)<r+\varepsilon$ definitively, so that

$$
\limsup _{k \rightarrow \infty} \rho\left(x_{k}, y\right) \leq r+\varepsilon .
$$

This holds for any $\varepsilon>0$, hence

$$
\limsup _{k \rightarrow \infty} \rho\left(x_{k}, y\right) \leq r .
$$

We now want to show that

$$
\liminf _{k \rightarrow \infty} \rho\left(x_{k}, y\right) \geq r,
$$

which will imply $\rho\left(x_{k}, y\right) \rightarrow \rho(x, y)$. Let again $r=\rho(x, y)$ and $\varepsilon>0$; then $x \notin B\left(y, r-\frac{\varepsilon}{2}\right)$. By (H2) (b),

$$
\overline{B(y, r-\varepsilon)} \subset B\left(y, r-\frac{\varepsilon}{2}\right),
$$

hence (denoting the complement of $A$ with $\left.A^{c}\right) x$ belongs to $\overline{B(y, r-\varepsilon)}^{c}$; since this is an open set, there exists $B(x, \eta) \subset \overline{B(y, r-\varepsilon)}^{c}$; hence $x_{k} \in \overline{B(y, r-\varepsilon)}^{c}$ definitively, which means that $\rho\left(x_{k}, y\right) \geq r-\varepsilon$ definitively, and

$$
\liminf _{k \rightarrow \infty} \rho\left(x_{k}, y\right) \geq r-\varepsilon \text {. }
$$

This holds for any $\varepsilon>0$, so (10) follows.

Conversely, assume now the continuity of $\rho$, and let us prove (H2). Let $y \in B(x, r)$, so that $\rho(x, y)<r$. Since $\rho$ is continuous, there exists $B\left(y, r^{\prime}\right)$ such that for any $z \in B\left(y, r^{\prime}\right)$ we have $\rho(x, z)<r$; hence $B\left(y, r^{\prime}\right) \subset B(x, r)$ and $B(x, r)$ is open, that is (H2) (a) holds.

Let now $y \in \overline{B(x, r)}$; since we already know that balls are open, as noted above this means that $y_{k} \rightarrow y$ for some sequence $\left\{y_{k}\right\} \subset B(x, r)$. Hence $\rho\left(y_{k}, x\right)<r$ and $\limsup _{k \rightarrow \infty} \rho\left(y_{k}, x\right) \leq r$. However, $\rho$ is continuous, so

$$
\limsup _{k \rightarrow \infty} \rho\left(y_{k}, x\right)=\rho(y, x),
$$

which means that $y \in\{z: \rho(z, x) \leq r\}$, which is (H2) (b).

The next property, which involves both $\rho$ and the measure $\mu$, tells us that also even when estimating Hölder norms, zero measure sets are negligible. 
Proposition 5 (i) Let $A, E \subset \Omega, A$ open and $E$ of measure zero. Then $\overline{A \backslash E}=$ $\bar{A}$.

(ii) Let $f: A \backslash E \rightarrow \mathbb{R}$ with $A, E$ as above, and $f$ such that, for some $\alpha, C>0$

$$
|f(x)-f(y)| \leq C \rho(x, y)^{\alpha}
$$

for any $x, y \in A \backslash E$. Then $f$ can be continuously extended to $\bar{A}$ in such a way that (11) holds for any $x, y \in \bar{A}$.

Proof. (i) Let $x \in \bar{A}$ and $\left\{x_{k}\right\} \subset A$ such that $x_{k} \rightarrow x$. Since $A$ is open, for any $k$ there exists $r_{k}>0$ such that $B\left(x_{k}, r_{k}\right) \subset A$. It is not restrictive to assume that $r_{k} \rightarrow 0$. For any $k$ there exists $y_{k} \in B\left(x_{k}, r_{k}\right) \backslash E$; otherwise $E$ would contain a ball, which by (H6) has positive measure. By (5), $x \in \Omega_{n}$ for some $n$; then, by (6) the sequence $\left\{x_{k}\right\}$ is definitively contained in $\Omega_{n+1}$; for the same reason the balls $B\left(x_{k}, r_{k}\right)$ are definitively contained in $\Omega_{n+2}$, hence we can apply the quasitriangle inequality (7) writing

$$
\rho\left(y_{k}, x\right) \leq B_{n+2}\left(\rho\left(y_{k}, x_{k}\right)+\rho\left(x_{k}, x\right)\right) \leq B_{n+2}\left(r_{k}+\rho\left(x_{k}, x\right)\right) \rightarrow 0
$$

for $k \rightarrow \infty$, so $y_{k} \rightarrow x$. Since $y_{k} \in A \backslash E$, this implies $x \in \overline{A \backslash E}$ and we are done.

(ii) Since $\rho$ is continuous, (11) implies that $f$ is uniformly continuous on $A \backslash E$, hence it can be continuously extended to $\overline{A \backslash E}$ in such a way that (11) still holds. By point (i) $\overline{A \backslash E}=\bar{A}$, so (ii) is proved.

\section{Dyadic cubes in a locally homogeneous space}

Throughout this paper, until Section 8 , we will assume that $\left(\Omega,\left\{\Omega_{n}\right\}_{n=1}^{\infty}, \rho, \mu\right)$ be a locally homogeneous space.

The construction of dyadic cubes, which has been anticipated in the introduction, is contained in the following:

Theorem 6 For any $n=1,2,3, \ldots$ there exists a collection of open sets

$$
\left\{Q_{\alpha}^{k} \subset \Omega, k=1,2,3 \ldots, \alpha \in I_{k}\right\}
$$

(where $I_{k}$ is a set of indices), positive constants $a_{0}, c_{0}, c_{1}, c_{2}, \delta \in(0,1)$ and a set $E \subset \Omega_{n}$ of zero measure, such that for any $k=1,2,3 \ldots$ we have:

(a) $\forall \alpha \in I_{k}$, each $Q_{\alpha}^{k}$ contains a ball $B\left(z_{\alpha}^{k}, a_{0} \delta^{k}\right)$;

(b) $\bigcup_{\alpha \in I_{k}} Q_{\alpha}^{k} \subset \Omega_{n+1}$;

(c) $\forall \alpha \in I_{k}, 1 \leq l \leq k$ there exists $Q_{\beta}^{l} \supseteq Q_{\alpha}^{k}$;

(d) $\forall \alpha \in I_{k}, \operatorname{diam}\left(Q_{\alpha}^{k}\right)<c_{1} \delta^{k}$ and $\overline{Q_{\alpha}^{k}} \subset B\left(z_{\alpha}^{k}, c_{1} \delta^{k}\right)$;

(e) $\ell \geq k \Longrightarrow \forall \alpha \in I_{k}, \beta \in I_{l}, Q_{\beta}^{\ell} \subset Q_{\alpha}^{k}$ or $Q_{\beta}^{\ell} \cap Q_{\alpha}^{k}=\emptyset$;

(f) $\Omega_{n} \backslash \bigcup_{\alpha \in I_{k}} Q_{\alpha}^{k} \subset E$; 
(g) $\forall \alpha \in I_{k}, x \in Q_{\alpha}^{k} \backslash E, j \geq 1$ there exists $Q_{\beta}^{j} \ni x$;

(h) $\mu\left(B(x, 2 r) \cap Q_{\alpha}^{k}\right) \leq c_{2} \mu\left(B(x, r) \cap Q_{\alpha}^{k}\right)$ for any $x \in Q_{\alpha}^{k} \backslash E, r>0$. More precisely, for these $x$ and $r$ we have:

$$
\mu\left(B(x, r) \cap Q_{\alpha}^{k}\right) \geq \begin{cases}c_{0} \mu(B(x, r)) & \text { for } r \leq \delta^{k} \\ c_{0} \mu\left(Q_{\alpha}^{k}\right) & \text { for } r>\delta^{k}\end{cases}
$$

Note that the cubes $Q_{\alpha}^{k}$ and all the constants depend on $n$, so we should write, more precisely

$$
\left\{Q_{\alpha}^{(n), k}\right\}_{\alpha \in I_{k}^{(n)}} ; \delta_{(n)} ; a_{0,(n)}, c_{0,(n)}, c_{1,(n)}, c_{2,(n)} .
$$

However, in order to simplify notation, we will skip the index $(n)$ whenever doing so does not create ambiguity. As will be apparent from the proof, the sequence of constants $\delta_{(n)}$ can be assumed nonincreasing.

The sets $Q_{\alpha}^{k}$ can be thought as dyadic cubes of sidelength $\delta^{k}$. Note that $k$ is a positive integer, so we are only considering small dyadic cubes.

The proof of Theorem [6 is not much more than a careful inspection and adaptation of some proofs contained in 16 . However, our iterative construction is a bit more involved because at every step $n$ the "universe" that we want to cover with our cubes enlarges. Moreover, in order to use the quasitriangle inequality, we need to know in advance that the points belong to some domain; this will be often proved by a tricky combined use of (6) and (7).

Proof of Theorem 6, first part. For a fixed $\Omega_{n}$, let $\delta>0$ to be fixed later, and let us perform the following iterative construction.

For $k=1$, let us fix a maximal collection of points $\left\{z_{\alpha}^{1}\right\}_{\alpha \in I_{1}} \subset \Omega_{n}$ such that

$$
\rho\left(z_{\alpha}^{k}, z_{\beta}^{k}\right) \geq \delta \text { for any } \alpha \neq \beta .
$$

By the maximality, we can say that for $x \in \Omega_{n}$ there exists $z_{\alpha}^{1}$ such that $\rho\left(z_{\alpha}^{1}, x\right)<\delta$, hence

$$
E_{1} \equiv \Omega_{n} \subseteq \bigcup_{\alpha \in I_{1}} B\left(z_{\alpha}^{1}, \delta\right) \equiv E_{2}
$$

For $k=2$, let us fix a maximal collection of points $\left\{z_{\alpha}^{2}\right\}_{\alpha \in I_{2}} \subset E_{2}$ such that

$$
\rho\left(z_{\alpha}^{2}, z_{\beta}^{2}\right) \geq \delta^{2} \text { for any } \alpha \neq \beta \text {. }
$$

By the maximality, we can say that for any $x \in E_{2}$ there exists $z_{\alpha}^{2}$ such that $\rho\left(z_{\alpha}^{1}, x\right)<\delta^{2}$, hence

$$
E_{2} \subseteq \bigcup_{\alpha \in I_{2}} B\left(z_{\alpha}^{2}, \delta^{2}\right) \equiv E_{3} .
$$

Continuing this way, we build a family of points $\left\{z_{\alpha}^{k}\right\}_{\alpha \in I_{k}}$ for $k=1,2,3, \ldots$, and a family of sets $E_{1} \subseteq E_{2} \subseteq E_{3} \subseteq \ldots$. We are going to show that it is possible 
to choose $\delta$ small enough so that

$$
\bigcup_{k=1}^{\infty} E_{k} \subset \Omega_{n+1} .
$$

Namely: $E_{1}=\Omega_{n} \subset \Omega_{n+1}$ and, by definition of $E_{2}$ and (6) $E_{2} \subset \Omega_{n+1}$ as soon as

$$
\delta<2 \varepsilon_{n} .
$$

Let now $y \in E_{3}$. Then there exists $z_{\alpha}^{2} \in E_{2}$ such that $\rho\left(y, z_{\alpha}^{2}\right)<\delta^{2}$ and there exists $z_{\beta}^{1} \in \Omega_{n}$ such that $\rho\left(z_{\alpha}^{2}, z_{\beta}^{1}\right)<\delta$. Since $E_{2} \subset \Omega_{n+1}$, we have $y \in \Omega_{n+2}$ (that is $E_{3} \subset \Omega_{n+2}$ ) as soon as

$$
\delta^{2}<2 \varepsilon_{n+1} .
$$

Under this assumption we can write

$$
\rho\left(y, z_{\beta}^{1}\right) \leq B_{n+2}\left(\rho\left(y, z_{\alpha}^{2}\right)+\rho\left(z_{\alpha}^{2}, z_{\beta}^{1}\right)\right) \leq B_{n+2}\left(\delta^{2}+\delta\right) .
$$

Then, under the further assumption

$$
B_{n+2}\left(\delta^{2}+\delta\right) \leq 2 \varepsilon_{n}
$$

we can conclude that $E_{3} \subset \Omega_{n+1}$ (which strengthen the previous conclusion $E_{3} \subset \Omega_{n+2}$ ).

This idea can be iterated showing that for any $y \in E_{N}, N=2,3,4 \ldots$ there exists $x \in \Omega_{n}$ such that

$$
\begin{aligned}
\rho(x, y) & \leq B_{n+2}\left[\delta+B_{n+2}\left[\delta^{2}+B_{n+2}\left[\delta^{3}+\ldots+B_{n+2}\left[\delta^{N-1}+\delta^{N}\right]\right]\right]\right] \\
& \leq \sum_{k=1}^{N}\left(B_{n+2} \delta\right)^{k} \leq \frac{\delta B_{n+2}}{1-\delta B_{n+2}}<2 \delta B_{n+2}
\end{aligned}
$$

provided

$$
\delta<1 /\left(2 B_{n+2}\right)
$$

If, moreover,

$$
\delta<\varepsilon_{n} / B_{n+2},
$$

we can conclude (13). Choosing $\delta$ small enough to fulfill conditions (14)-(19) we are done.

Note that, for any $k=2,3 \ldots, \alpha \in I_{k}$,

$$
z_{\alpha}^{k} \in E_{k}=\bigcup_{\beta \in I_{k-1}} B\left(z_{\beta}^{k-1}, \delta^{k-1}\right) \supseteq E_{k-1}
$$

hence for any $z_{\alpha}^{k}$ there exists $\beta \in I_{k-1}$ such that

$$
\rho\left(z_{\alpha}^{k}, z_{\beta}^{k-1}\right)<\delta^{k-1}
$$


Moreover, for any $k=1,2,3 \ldots$,

$$
\rho\left(z_{\alpha}^{k}, z_{\beta}^{k}\right) \geq \delta^{k} \text { for any } \alpha \neq \beta \text {. }
$$

After this preliminary construction we pause for a moment our proof, and give the following

Definition $7 A$ tree is a partial ordering $\leq$ of the set of all ordered pairs $(k, \alpha)$ $\left(k=1,2,3, \ldots, \alpha \in I_{k}\right)$ which satisfies:

(T1) $(k, \alpha) \leq(l, \beta) \Rightarrow k \geq l$.

(T2) For each $(k, \alpha)$ and $1 \leq l \leq k$ there exists a unique $\beta$ such that $(k, \alpha) \leq$ $(l, \beta)$.

(T3) $(k, \alpha) \leq(k-1, \beta) \Longrightarrow \rho\left(z_{\alpha}^{k}, z_{\beta}^{k-1}\right)<\delta^{k-1}$.

(T4) $\rho\left(z_{\alpha}^{k}, z_{\beta}^{k-1}\right)<\left(2 B_{n}\right)^{-1} \delta^{k-1} \Longrightarrow(k, \alpha) \leq(k-1, \beta)$.

It is not restrictive to assume

$$
B_{n} \geq 2,
$$

as we will do in the following; hence the constant $\left(2 B_{n}\right)^{-1}$ appearing in the definition is $\leq 1$.

This definition is the same given in [16], except for the restriction that our integers $k, l$ are positive. Moreover, our tree also depends on $n$ (through the points $z_{\alpha}^{k}$ ). As proved in [16, Lemma 13], there exists at least one tree (for each integer $n$ ). Actually, the same proof applies in view of (21), (22).

As in [16, we can now define the dyadic cubes:

Definition 8 For a fixed integer $n$, fix a tree, and let $a_{0} \in(0,1)$ be a small constant to be determined. For $k=1,2,3, \ldots, \alpha \in I_{k}$, set

$$
Q_{\alpha}^{k}=\bigcup_{(l, \beta) \leq(k, \alpha)} B\left(z_{\beta}^{l}, a_{0} \delta^{l}\right) .
$$

Proof of Theorem 6, second part. By definition, each $Q_{\alpha}^{k}$ is an open set and (a) holds. Since

$$
B\left(z_{\beta}^{l}, a_{0} \delta^{l}\right) \subseteq B\left(z_{\beta}^{l}, a_{0} \delta\right)
$$

we have

$$
Q_{\alpha}^{k} \subseteq \bigcup_{(l, \beta) \leq(k, \alpha)} B\left(z_{\beta}^{l}, a_{0} \delta\right)
$$

Since $z_{\beta}^{l} \in \bigcup_{k=1}^{\infty} E_{k} \subset \Omega_{n+1}$, choosing $a_{0}$ such that

$$
a_{0} \delta<\varepsilon_{n+1}
$$


we read that $Q_{\alpha}^{k} \subset \Omega_{n+2}$. Let $y \in Q_{\alpha}^{k}$ and $z_{\beta}^{l}$ such that $\rho\left(y, z_{\beta}^{l}\right)<a_{0} \delta$.By (17), there exists $x \in \Omega_{n}$ such that $\rho\left(x, z_{\beta}^{l}\right)<2 \delta B_{n+2}$, hence

$$
\rho(x, y) \leq B_{n+2}\left(\rho\left(x, z_{\beta}^{l}\right)+\rho\left(y, z_{\beta}^{l}\right)\right) \leq B_{n+2}\left(2 \delta B_{n+2}+a_{0} \delta\right)
$$

so we can conclude that $y \in \Omega_{n+1}$ provided

$$
\delta\left(2 B_{n+2}^{2}+a_{0} B_{n+2}\right)<2 \varepsilon_{n} .
$$

It is now useful to choose $a_{0}=\delta$; this implies that all the conditions we will write on $a_{0}$ and $\delta$ simply ask that $\delta$ be small enough in terms of the constants $\varepsilon_{n}, B_{n}$, so that all these conditions can be simultaneously satisfied. Nevertheless, we will keep using both the symbols $a_{0}$ and $\delta$, to stress the different roles of these constants.

Under assumptions (25)-(26), we conclude $Q_{\alpha}^{k} \subset \Omega_{n+1}$, that is (b) holds.

From the definition (24) we also have the monotonicity of dyadic cubes:

$$
(l, \beta) \leq(k, \alpha) \Longrightarrow Q_{\beta}^{l} \subseteq Q_{\alpha}^{k} .
$$

By (T2) and (27) we immediately have (c).

As in [16, (3.13)] we can prove that

$$
(l, \beta) \leq(k, \alpha) \Longrightarrow \rho\left(z_{\beta}^{l}, z_{\alpha}^{k}\right) \leq 2 B_{n+1} \delta^{k}
$$

provided

$$
\delta<\left(2 B_{n}\right)^{-1} .
$$

This implies (d) since, for any $x, y \in Q_{\alpha}^{k}, x \in B\left(z_{\beta}^{l}, a_{0} \delta^{l}\right), y \in B\left(z_{\gamma}^{h}, a_{0} \delta^{h}\right)$ for some $(l, \beta) \leq(k, \alpha),(h, \gamma) \leq(k, \alpha)$ we can write

$$
\begin{aligned}
\rho(x, y) & \leq B_{n+1}\left[\rho\left(x, z_{\beta}^{l}\right)+\rho\left(y, z_{\beta}^{l}\right)\right] \\
& \leq B_{n+1}\left[a_{0} \delta^{l}+B_{n+1}\left[\rho\left(y, z_{\gamma}^{h}\right)+\rho\left(z_{\beta}^{l}, z_{\gamma}^{h}\right)\right]\right] \\
& \leq B_{n+1}\left[a_{0} \delta^{l}+B_{n+1}\left[a_{0} \delta^{h}+B_{n+1}\left[\rho\left(z_{\beta}^{l}, z_{\alpha}^{k}\right)+\rho\left(z_{\alpha}^{k}, z_{\gamma}^{h}\right)\right]\right]\right] \\
& \leq B_{n+1}\left[a_{0} \delta^{l}+B_{n+1}\left[a_{0} \delta^{h}+B_{n+1}\left[2 B_{n+1} \delta^{k}+2 B_{n+1} \delta^{k}\right]\right]\right]
\end{aligned}
$$

where the last inequality follows by (28). Since $l, h \geq k$ this implies (since $\left.a_{0}<1\right)$

$$
\rho(x, y) \leq \delta^{k}\left[B_{n+1}+B_{n+1}^{2}+4 B_{n+1}^{4}\right]
$$

which gives (d) with

$$
c_{1}=7 B_{n+1}^{4} .
$$

Note that with this choice of $c_{1}$ we also have $\overline{Q_{\alpha}^{k}} \subset B\left(z_{\alpha}^{k}, c_{1} \delta^{k}\right)$.

In order to prove (e), we can now proceed proving, as [16, Lemma 15]:

$$
\text { If } Q_{\alpha}^{k} \cap Q_{\beta}^{k} \neq \emptyset \text { then } \alpha=\beta \text {. }
$$


Indeed, the same proof of [16, Lemma 15] applies, in view of (28), (b), (22). More precisely, (31) holds provided we choose $a_{0}$ and $\delta$ small enough so that

$$
\delta+a_{0}<\left(2 B_{n+1}\right)^{-3} .
$$

With (31) in hand let us show that (e) holds. Let $l \geq k \geq 1, Q_{\beta}^{\ell} \cap Q_{\alpha}^{k} \neq \emptyset$, and choose $\gamma$ such that $(l, \beta) \leq(k, \gamma)$ (this is possible by (T2)); then $Q_{\beta}^{l} \subseteq Q_{\gamma}^{k}$ which, together with $Q_{\beta}^{\ell} \cap Q_{\alpha}^{k} \neq \emptyset$ implies $Q_{\alpha}^{k} \cap Q_{\gamma}^{k} \neq \emptyset$. By (31) then $\alpha=\gamma$, that is $Q_{\beta}^{l} \subseteq Q_{\alpha}^{k}$ which gives (e).

Let us come to the proof of (f). Fix $k \geq 1$ and let

$$
F_{k}=\bigcup_{\alpha \in I_{k}} Q_{\alpha}^{k}
$$

Fix $x \in \Omega_{n}=E_{1}$; since $E_{1} \subseteq E_{2} \subseteq E_{3} \subseteq \ldots$, by (20),

$$
\forall h \geq 1 \exists z_{\alpha}^{h} \text { such that } \rho\left(x, z_{\alpha}^{h}\right)<\delta^{h} .
$$

By (b), for any $h \geq k$ we have

$$
B\left(z_{\alpha}^{h}, a_{0} \delta^{h}\right) \subseteq Q_{\alpha}^{h} .
$$

By (c) there exists $Q_{\beta}^{k} \supseteq Q_{\alpha}^{h}$, hence

$$
B\left(z_{\alpha}^{h}, a_{0} \delta^{h}\right) \subseteq Q_{\alpha}^{h} \subseteq Q_{\beta}^{k} \subseteq F_{k} \subset \Omega_{n+1} .
$$

By the triangle inequality,

$$
B\left(z_{\alpha}^{h}, a_{0} \delta^{h}\right) \subseteq B\left(x, B_{n+1}\left(1+a_{0}\right) \delta^{h}\right) \equiv B .
$$

In turn,

$$
B\left(x, B_{n+1}\left(1+a_{0}\right) \delta^{h}\right) \subseteq B\left(z_{\alpha}^{h}, B_{n+1}\left(B_{n+1}\left(1+a_{0}\right) \delta^{h}+\delta^{h}\right)\right) .
$$

For $h$ large enough we have

$$
B_{n+1}\left(B_{n+1}\left(1+a_{0}\right) \delta^{h}+\delta^{h}\right) \leq 3 B_{n+1}^{2} \delta^{h} \leq \varepsilon_{n},
$$

and the local doubling condition (8) implies

$$
\mu\left(B\left(z_{\alpha}^{h}, a_{0} \delta^{h}\right)\right) \geq c \mu(B)
$$

for some constant $c>0$ depending on $n$ (once we have fixed $\delta$ and $a_{0}$ ). By (34) and (35) the last inequality gives

$$
\frac{\mu\left(F_{k} \cap B\right)}{\mu(B)} \geq \frac{\mu\left(B\left(z_{\alpha}^{h}, a_{0} \delta^{h}\right) \cap B\right)}{\mu(B)}=\frac{\mu\left(B\left(z_{\alpha}^{h}, a_{0} \delta^{h}\right)\right)}{\mu(B)} \geq c>0
$$

and $h$ large enough. Letting $h \rightarrow+\infty$ we find that

$$
\limsup _{r \rightarrow 0} \frac{\mu\left(F_{k} \cap B(x, r)\right)}{\mu(B(x, r))} \geq c>0 \quad \forall x \in \Omega_{n}, k=1,2,3 \ldots
$$


By Lebesgue's theorem on differentiation of the integral, $\mu\left(\Omega_{n} \backslash F_{k}\right)=0$. Letting

$$
E=\bigcup_{k=1}^{\infty}\left(\Omega_{n} \backslash F_{k}\right)
$$

we have (f).

To prove $(\mathrm{g})$ we need a refinement of the argument used in the above proof of (f). Since $\Omega_{n}=E_{1} \subseteq E_{2} \subseteq E_{3} \subseteq \ldots$, by (20) for any $x \in E_{N}$ we have that:

$$
\forall h \geq N \exists z_{\alpha}^{h} \text { such that } \rho\left(x, z_{\alpha}^{h}\right)<\delta^{h}
$$

while for any $h \geq k$ (34) and (35) still hold. Hence we can prove as above that

$$
\mu\left(E_{h} \backslash F_{k}\right)=0 \text { for any } k, h \geq 1 .
$$

Let $F$ be the null set given by $\bigcup_{h, k \geq 1}\left(E_{h} \backslash F_{k}\right)$. Then fix a dyadic cube $Q_{\alpha}^{k}$ and pick a point $x \in Q_{\alpha}^{k} \backslash F$. Since $x \in Q_{\alpha}^{k}$, there exists $B\left(z_{\beta}^{h}, a_{0} \delta^{h}\right) \ni x$ for some $h \geq k$; since

$$
B\left(z_{\beta}^{h}, a_{0} \delta^{h}\right) \subset B\left(z_{\beta}^{h}, \delta^{h}\right) \subset E_{h},
$$

this means that $x \in E_{h}$; since $x \notin F$, (38) implies that for any $l \geq 1$ the point $x$ belongs to some $Q_{\beta}^{l}$, which is (g). Clearly, the fact that the null set $F$ appearing in the proof of this point is possibly different from the null set $E$ appearing in the proof of point (f) is immaterial, since we can always relabel $E$ the union of the two.

To prove (h), let $x \in Q_{\alpha}^{k} \backslash F$ (with $F$ as above) and $r>0$. We need to establish a lower bound on $\mu\left(B(x, r) \cap Q_{\alpha}^{k}\right)$; let us distinguish two cases:

(i) $r \leq \delta^{k}$. Let $j \geq k$ such that $\delta^{j+1}<r \leq \delta^{j}$ and let $Q_{\beta}^{j+2}$ a cube containing $x$ (by (g) it certainly exists). By (e), $Q_{\beta}^{j+2} \subset Q_{\alpha}^{k}$ while by (d), $\operatorname{diam}\left(Q_{\beta}^{j+2}\right) \leq$ $c_{1} \delta^{j+2}$. Then $Q_{\beta}^{j+2} \subset B(x, r)$, since, for $y \in Q_{\beta}^{j+2}$,

$$
\rho(x, y) \leq B_{n+1}\left[\rho\left(x, z_{\beta}^{j+2}\right)+\rho\left(y, z_{\beta}^{j+2}\right)\right] \leq 2 B_{n+1} c_{1} \delta^{j+2} \leq \delta^{j+1}<r
$$

provided $\delta$ is small enough so that

$$
2 B_{n+1} c_{1} \delta \leq 1 .
$$

Therefore

$$
\begin{aligned}
\mu\left(B(x, r) \cap Q_{\alpha}^{k}\right) & \geq \mu\left(Q_{\beta}^{j+2}\right) \geq \mu\left(B\left(z_{\beta}^{j+2}, a_{0} \delta^{j+2}\right)\right) \\
& \geq c_{0} \mu\left(B\left(x, \delta^{j}\right)\right) \geq c_{0} \mu(B(x, r))
\end{aligned}
$$

where the up to last inequality follows by the local doubling condition (8), with a constant $c_{0}$ only depending on $n$. 
(ii) $r>\delta^{k}$. Let $Q_{\beta}^{k+1} \ni x$ (by point (g) it certainly exists). Since $\operatorname{diam}\left(Q_{\beta}^{k+1}\right) \leq$ $c_{1} \delta^{k+1}$

$$
Q_{\beta}^{k+1} \subset B\left(x, c_{1} \delta^{k+1}\right) \subset B(x, r)
$$

as soon as

$$
c_{1} \delta<1
$$

hence, by point (a),

$$
\mu\left(B(x, r) \cap Q_{\alpha}^{k}\right) \geq \mu\left(Q_{\beta}^{k+1}\right) \geq \mu\left(B\left(z_{\beta}^{k+1}, a_{0} \delta^{k+1}\right)\right)
$$

while, since $z_{\beta}^{k+1} \in Q_{\alpha}^{k}$ and $\operatorname{diam}\left(Q_{\alpha}^{k}\right) \leq c_{1} \delta^{k}$,

$$
\mu\left(B\left(z_{\beta}^{k+1}, c_{1} \delta^{k}\right)\right) \geq \mu\left(Q_{\alpha}^{k}\right) .
$$

To conclude (12), which immediately give (h), we have to apply the local doubling condition, to say that

$$
\mu\left(B\left(z_{\beta}^{k+1}, c_{1} \delta^{k}\right)\right) \leq c_{0} \mu\left(B\left(z_{\beta}^{k+1}, a_{0} \delta^{k+1}\right)\right) .
$$

This is possible, once we have (at last) fixed $\delta$, with some constant depending on $\delta$, and therefore on $n$. Hence Theorem 6 is proved.

Finally, note that in our iterative construction, at every step $n$ we can always choose the number $\delta_{(n)}$ less than or equal to the number $\delta_{(n-1)}$ chosen at the previous step. Hence the sequence $\delta_{(n)}$ can be assumed to be nonincreasing.

Remark 9 In the previous proof the reader could be confused by the number of conditions we have imposed on $\delta$ and the other constants. So, let us summarize the logical line of this procedure. First, we can assume without loss of generality that the parameters $\varepsilon_{n}, B_{n}$ of $\Omega_{n}$ satisfy the following:

$$
\begin{aligned}
B_{n+1} & \geq B_{n} \geq 2 \text { for every } n \\
\varepsilon_{n+1} & \leq \varepsilon_{n} \leq \frac{1}{2} \text { for every } n .
\end{aligned}
$$

Then we have chosen

$$
c_{1}=7 B_{n+1}^{4}
$$

and $a_{0}=\delta$, where $\delta$ has to satisfy conditions (14), (15), (16), (18), (19), (25), (26), (29), (32), (39), (40), and also (43), which will be used in the proof of Lemma 12, With some patience one can check that a possible choice is

$$
\delta=\frac{1}{2} \min \left(\varepsilon_{n+1}, \frac{\varepsilon_{n}}{4 B_{n+2}^{2}}, \frac{1}{14 B_{n+1}^{5}}\right) .
$$

After $\delta$ has been fixed, the constants $c_{0}, c_{2}$ can be determined in terms of $\delta$ and $C_{n}$. 
Point (h) of the above theorem means that each set $Q_{\alpha}^{k} \backslash E$ is a space of homogeneous type. It is useful to reinforce the previous statement with the following:

Proposition 10 For each $\Omega_{n}, k$ and $\alpha \in I_{k}$, the set $Q_{\alpha}^{k}$ is a space of homogeneous type.

Here and in the following, whenever we will write that a set $S \subset \Omega$ is a space of homogeneous type we will mean that $(S, \rho, d \mu)$ is a space of homogeneous type, with respect to the same $\rho$ and $\mu$ already defined in $\Omega$.

Proof. The only point to prove is that if $x \in Q_{\alpha}^{k} \cap E$ (where $E$ is like in Theorem 6) then

$$
\mu\left(B(x, 2 r) \cap Q_{\alpha}^{k}\right) \leq c \mu\left(B(x, r) \cap Q_{\alpha}^{k}\right) \text { for any } r>0 .
$$

Pick $y \in B(x, \varepsilon r) \cap\left(Q_{\alpha}^{k} \backslash E\right)$ for some small $\varepsilon$ to be fixed later. Such $y$ certainly exists, otherwise $E$ would contain the open set $B(x, \varepsilon r) \cap Q_{\alpha}^{k}$, which by (8) has positive measure, while $E$ has zero measure.

Since $x \in Q_{\alpha}^{k} \subset \Omega_{n+1}$, for $r \leq \varepsilon_{n+1}$ we have $B(x, 2 r) \subset \Omega_{n+2}$ and we can prove

$$
B(x, 2 r) \subset B\left(y, c_{1} r\right)
$$

with $c_{1}=(2+\varepsilon) B_{n+2}$. Analogously,

$$
B\left(y, c_{2} r\right) \subset B(x, r)
$$

provided $\left(\varepsilon+c_{2}\right) B_{n+2}<1$. Hence (41), (42) hold for suitable constants $c_{2}<$ $1<c_{1}$ and $\varepsilon$ small enough (depending on $n$ but not on $r$ ), while by point (h) of Theorem [6, since $y \in Q_{\alpha}^{k} \backslash E$ we have

$$
\mu\left(B\left(y, c_{1} r\right) \cap Q_{\alpha}^{k}\right) \leq c \mu\left(B\left(y, c_{2} r\right) \cap Q_{\alpha}^{k}\right)
$$

for some constant $c$ depending on $c_{1}, c_{2}$ and any $r>0$. So we conclude

$$
\mu\left(B(x, 2 r) \cap Q_{\alpha}^{k}\right) \leq c \mu\left(B(x, r) \cap Q_{\alpha}^{k}\right) \text { for any } r \leq \varepsilon_{n+1} .
$$

Let now $r>\varepsilon_{n+1}$. Pick $y \in B\left(x, \varepsilon_{n+1} / 2 B_{n+2}\right) \cap\left(Q_{\alpha}^{k} \backslash E\right)$. By (g), for any $h$ there exists $Q_{\beta}^{h} \ni y$. Since $\operatorname{diam}\left(Q_{\beta}^{h}\right) \leq c_{1} \delta^{h}$, for $z \in Q_{\beta}^{h}$ we have

$$
\rho(z, x) \leq B_{n+2}(\rho(z, y)+\rho(y, x)) \leq B_{n+2} c_{1} \delta^{h}+\frac{\varepsilon_{n+1}}{2}<\varepsilon_{n+1}
$$

for $h$ large enough. Let $h_{0}$ the minimum integer $\geq k$ such that this is true, so that $Q_{\beta}^{h_{0}} \subset B\left(x, \varepsilon_{n+1}\right)$. Hence

$$
\mu\left(B(x, r) \cap Q_{\alpha}^{k}\right) \geq \mu\left(Q_{\beta}^{h_{0}}\right)
$$

while $\mu\left(B(x, 2 r) \cap Q_{\alpha}^{k}\right) \leq \mu\left(Q_{\alpha}^{k}\right)$. The desired conclusion follows since $\mu\left(Q_{\alpha}^{k}\right)$ and $\mu\left(Q_{\beta}^{h_{0}}\right)$ are comparable. (See the last part of the proof of Theorem 6 ). So we are done. 
By Theorem[6] point (f), we know that each family of cubes $\left\{Q_{\alpha}^{k}\right\}_{\alpha \in I_{k}}$ covers $\Omega_{n} \backslash E$. Since the cubes $Q_{\alpha}^{k}$ are open and disjoint sets, it is reasonable that they cannot generally cover the whole $\Omega_{n}$ (if, for instance, $\Omega_{n}$ is a connected set, this is impossible). On the other hand, from the proof of the theorem we can read the following fact:

Proposition 11 For any $\Omega_{n}$ and any $k=1,2,3, \ldots$, the closure of $\bigcup_{\alpha \in I_{k}} Q_{\alpha}^{k}$ covers $\Omega_{n}$.

Proof. Let $x \in \Omega_{n}$. By (33), $\forall h \geq 1 \exists z_{\alpha_{h}}^{h}$ such that $\rho\left(x, z_{\alpha_{h}}^{h}\right)<\delta^{h}$. Hence the sequence $\left\{z_{\alpha_{k}}^{k}\right\}_{k=1}^{\infty}$ converges to $x$. Moreover, the point $z_{\alpha_{1}}^{1}$ belongs to $Q_{\alpha_{1}}^{1} \subset \cup_{\beta \in I_{1}} Q_{\beta}^{1}$; the point $z_{\alpha_{2}}^{2}$ belongs to a cube $Q_{\alpha_{2}}^{2}$ which is contained in some parent cube $Q_{\gamma}^{1} \subset \cup_{\beta \in I_{1}} Q_{\beta}^{1}$, and so on. Hence the whole sequence is contained in $\cup_{\beta \in I_{1}} Q_{\beta}^{1}$, which means that $x$ belongs to the closure of $\cup_{\beta \in I_{1}} Q_{\beta}^{1}$. With the same reasoning we can say that for any positive integer $h$ the sequence $\left\{z_{\alpha_{k}}^{k}\right\}_{k=h}^{\infty}$ is contained in $\cup_{\beta \in I_{h}} Q_{\beta}^{h}$, hence $x$ belongs to the closure of $\cup_{\beta \in I_{h}} Q_{\beta}^{h}$ for any $h=1,2,3 \ldots$

The next question we pose is: how many cubes form each family $\left\{Q_{\alpha}^{k}\right\}_{\alpha \in I_{k}}$ ? We expect them to be finitely many, since they are contained in $\Omega_{n+1}$, which is bounded, they are pairwise disjoint and have essentially the same diameter. A formal proof of this fact requires some care. We first need the following lemma, which will be useful also other times.

Lemma 12 For any $k=1,2,3, \ldots$ there exists $c_{n, k}>0$ such that

$$
\inf _{z \in \Omega_{n}} \mu\left(B\left(z, a_{0} \delta^{k}\right)\right) \geq c_{n, k}
$$

where $\delta$ and $a_{0}$ are as in Theorem 6 .

Proof. Since $\bar{\Omega}_{n}$ is compact (see assumption (H4)), there exists a finite number of points $z_{1}, \ldots, z_{N} \in \bar{\Omega}_{n}$ such that

$$
\bar{\Omega}_{n} \subset \bigcup_{i=1}^{N} B\left(z_{i}, a_{0} \delta^{k}\right) .
$$

Let now $z$ be any point of $\Omega_{n}$; there exists $i_{0}$ such that $z \in B\left(z_{i_{0}}, a_{0} \delta^{k}\right)$. On the other hand, for any pair of nondisjoint balls of radius $r$ and centers $z, z_{i_{0}}$, we have the inclusion $B\left(z_{i_{0}}, r\right) \subset B\left(z, B_{n+1}\left(2 B_{n+1}+1\right) r\right)$. Assuming

$$
\left(2 B_{n+1}+1\right) a_{0} \delta \leq 2 \varepsilon_{n}
$$

we have, by the doubling condition (8)

$$
\mu\left(B\left(z, a_{0} \delta^{k}\right)\right) \geq c \mu\left(B\left(z,\left(2 B_{n}+1\right) a_{0} \delta^{k}\right)\right) \geq c \mu\left(B\left(z_{i_{0}}, a_{0} \delta^{k}\right)\right) \geq c \varepsilon \equiv c_{n, k}
$$

having set

$$
\varepsilon=\min _{i=1,2, \ldots, N} \mu\left(B\left(z_{i}, a_{0} \delta^{k}\right)\right)
$$


Proposition 13 For each $k=1,2,3, \ldots$, the family $\left\{Q_{\alpha}^{k}\right\}_{\alpha \in I_{k}}$ is finite.

Proof. Since $\bigcup_{\alpha \in I_{k}} Q_{\alpha}^{k} \subset \Omega_{n+1}$, we have

$$
\mu\left(\bigcup_{\alpha \in I_{k}} Q_{\alpha}^{k}\right) \leq \mu\left(\Omega_{n+1}\right)<\infty
$$

(recall that any $\Omega_{n+1}$ has finite measure, as noted after definition 1). Since the $Q_{\alpha}^{k}$ 's are pairwise disjoint and by Theorem [6] point (a), $Q_{\alpha}^{k} \supset B\left(z_{\alpha}^{k}, a_{0} \delta^{k}\right)$,

$$
\mu\left(\bigcup_{\alpha \in I_{k}} Q_{\alpha}^{k}\right)=\sum_{\alpha \in I_{k}} \mu\left(Q_{\alpha}^{k}\right) \geq \sum_{\alpha \in I_{k}} \mu\left(B\left(z_{\alpha}^{k}, a_{0} \delta^{k}\right)\right)
$$

where the last sum, by the previous Lemma, is an infinite quantity unless $I_{k}$ is finite. Therefore $I_{k}$ is finite.

The finiteness of the covering $\left\{Q_{\alpha}^{k}\right\}_{\alpha \in I_{k}}$ of $\Omega_{n}$ at any scale $k$ is interesting for the following consequence:

Corollary $\mathbf{1 4}$ For any $k=1,2,3, \ldots$ the set

$$
F_{k}=\bigcup_{\alpha \in I_{k}} Q_{\alpha}^{k}
$$

is a space of homogeneous type. The same conclusion holds for the union of any subfamily of $\left\{Q_{\alpha}^{k}\right\}_{\alpha \in I_{k}}$. The doubling constants depends on $n$ and $k$.

Proof. We have to prove that

$$
\mu\left(B(x, 2 r) \cap F_{k}\right) \leq c \mu\left(B(x, r) \cap F_{k}\right) \text { for any } r>0, x \in F_{k} .
$$

Let us first prove this inequality when $x \in F_{k} \backslash E$, where $E$ is the null set appearing in Theorem 6. So, let $x \in Q_{\alpha}^{k} \backslash E$ for some $\alpha \in I_{k}$ and let $r>0$. We will apply (12) in Theorem 6] distinguishing the cases $r \leq \delta^{k}$ and $r>\delta^{k}$.

When $r \leq \delta^{k}$, by the doubling condition (8) we have

$$
\begin{aligned}
\mu\left(B(x, r) \cap F_{k}\right) & \geq \mu\left(B(x, r) \cap Q_{\alpha}^{k}\right) \geq c_{0} \mu(B(x, r)) \\
& \geq \frac{c_{0}}{C_{n}} \mu(B(x, 2 r)) \geq \frac{c_{0}}{C_{n}} \mu\left(B(x, 2 r) \cap F_{k}\right) .
\end{aligned}
$$

When $r>\delta^{k}$

$$
\begin{aligned}
\mu\left(B(x, r) \cap F_{k}\right) & \geq \mu\left(B(x, r) \cap Q_{\alpha}^{k}\right) \geq c_{0} \mu\left(Q_{\alpha}^{k}\right) \\
& \geq c_{n, k} \mu\left(F_{k}\right) \geq c_{n, k} \mu\left(B(x, 2 r) \cap F_{k}\right)
\end{aligned}
$$

where in the up to last inequality we have used the fact that the $Q_{\alpha}^{k}$ are finitely many open sets, each of positive measure, while $\mu\left(F_{k}\right) \leq \mu\left(\Omega_{n+1}\right)<\infty$, so 
that for some constant $c$ depending on $n$ and $k$ (but not on $\alpha$ ), we can write $\mu\left(Q_{\alpha}^{k}\right) \geq c \mu\left(F_{k}\right)$.

If now $x \in F_{k} \cap E$, we can repeat the same reasoning used in the proof of Proposition 10 to show that (44) still holds. This completes the proof.

Summarizing several results proved so far, we can say that for any $n$ there exists a space of homogeneous type $F_{k}$, contained in $\Omega_{n+1}$ and essentially containing $\Omega_{n}$, in the sense that $\Omega_{n} \backslash E \subset F_{k}$ (by Theorem [6, f) and $\Omega_{n} \subset \bar{F}_{k}$ (by Proposition 11). In view of our applications to singular integrals, it is important to get a local and more quantitative version of this result. This is contained in the following theorem, which is the main result in this section. Since it involves different sets $\Omega_{n}$, here we have to add an index $n$ to the number $\delta$ and the cubes $Q_{\alpha}^{k}$.

Theorem 15 For every $n$ there exists $R_{n}>0$ such that for any $\bar{x} \in \Omega_{n}$ and $R \leq R_{n}$ there exists an open set $F$ such that:

(i) $F$ is a space of homogeneous type; its doubling constant depends on $n$ but not on $R$;

(ii) $B(\bar{x}, R) \backslash E \subset F \subset \Omega_{n+2}$ (with $\mu(E)=0$ );

(iii) $B(\bar{x}, R) \subset \bar{F}$;

(iv) diamF $\leq c R$ for some constant $c$ depending on $n$ but not on $R$;

(v) $\mu(F) \leq c \mu(B(\bar{x}, R))$ for some constant $c$ depending on $n$ but not on $R$.

The independence of the constants from $R$ will be precious when dealing with commutators of singular or fractional integrals with $V M O$ functions. Clearly, the whole $\Omega_{n}$ can be covered, for any $R \leq R_{n}$, by a finite number of balls $B\left(x_{i}, R\right)$, to which this theorem is applicable.

Remark 16 The reader could ask why we do not consider the set $\bar{F}$ (which satisfies the simple inclusions $B(\bar{x}, R) \subset \bar{F} \subset \Omega_{n+2}$ ) instead of $F$ (which does not exactly contain $B(\bar{x}, R)$ ). The problem with $\bar{F}$ is that, in our abstract context, it is not obvious how to prove that it is a space of homogeneous type, too.

Proof. Fix $\bar{x} \in \Omega_{n}$ and let $R_{n}=\delta_{(n)}^{k_{0}}$ for a $k_{0}$ to be chosen later, but such that $R_{n} \leq 2 \varepsilon_{n}$, hence $B(\bar{x}, R) \subset \Omega_{n+1}$. For $R \leq R_{n}$, pick $k \geq k_{0}$ such that $\delta_{(n)}^{k+1}<R \leq \delta_{(n)}^{k}$. For these $n$ and $k$, there exists $\alpha \in I_{k}^{(n)}$ such that (see Theorem 6)

$$
\bar{x} \in Q_{\alpha}^{(n), k} \subset B\left(z_{\alpha}^{(n), k}, c_{1,(n)} \delta_{(n)}^{k}\right) \subset \Omega_{n+1} .
$$

For any $y \in B(\bar{x}, R)$ we can write

$\rho\left(y, z_{\alpha}^{(n), k}\right) \leq B_{n+1}\left(\rho(y, \bar{x})+\rho\left(\bar{x}, z_{\alpha}^{(n), k}\right)\right)<B_{n+1}\left(\delta_{(n)}^{k}+c_{1,(n)} \delta_{(n)}^{k}\right) \equiv h_{n} \delta_{(n)}^{k}$

hence

$$
B(\bar{x}, R) \subset B\left(z_{\alpha}^{(n), k}, h_{n} \delta_{(n)}^{k}\right)
$$


with

$$
h_{n}=B_{n+1}\left(1+c_{1,(n)}\right) .
$$

Choose $k_{0}$ (and consequently $R_{n}$ ) so that $h_{n} \delta_{(n)}^{k_{0}} \leq 2 \varepsilon_{n}$, hence

$$
B\left(z_{\alpha}^{(n), k}, h_{n} \delta_{(n)}^{k}\right) \subset \Omega_{n+1}
$$

for any $k \geq k_{0}$, and so for any $R \leq R_{n}$. Since $\Omega_{n+1}$ is covered (up to a null set) by the union of all the dyadic cubes $Q_{\beta}^{(n+1), k}$, we can define the set

$$
F=\bigcup\left\{Q_{\beta}^{(n+1), k}: Q_{\beta}^{(n+1), k} \cap B\left(z_{\alpha}^{(n), k}, h_{n} \delta_{(n)}^{k}\right) \neq \emptyset\right\}
$$

and we immediately get

$$
B(\bar{x}, R) \backslash E \subset B\left(z_{\alpha}^{(n), k}, h_{n} \delta_{(n)}^{k}\right) \backslash E \subset F \subset \Omega_{n+2},
$$

that is (ii). Moreover, by Proposition 11 we also have $B(\bar{x}, R) \subset \bar{F}$, which is (iii).

By Corollary 14 $F$ is a space of homogeneous type. Note that, for the moment, we only know that its doubling constant depends on $n$ and $k$ (that is on $R$ ); we want to prove that it actually only depends on $n$.

Since

$$
\operatorname{diam} Q_{\beta}^{(n+1), k}<c_{1,(n+1)} \delta_{(n+1)}^{k} \leq c_{1,(n+1)} \delta_{(n)}^{k}
$$

(the sequence $\delta_{(n)}$ is nonincreasing) and each of the cubes defining $F$ intersects $B\left(z_{\alpha}^{(n), k}, h_{n} \delta_{(n)}^{k}\right)$, the quasitriangle inequality in $\Omega_{n+2}$ gives $\operatorname{diam} F \leq c \delta_{(n)}^{k}$ for some constant $c$ depending on $n$, that is (iv). Finally, since $B(\bar{x}, R) \supset$ $B\left(\bar{x}, \delta_{(n)}^{k+1}\right)$, repeated applications of the quasitriangle inequality in $\Omega_{n+2}$ give $F \subset B\left(\bar{x}, j_{n} R\right)$ for some constant $j_{n}$ dependent on $n$ but not on $R$. Shrinking if necessary the number $R_{n}$ (that is enlarging the integer $k_{0}$ ) we can assure that the local doubling condition in $\Omega_{n+2}$ is applicable to the ball $B\left(\bar{x}, j_{n} R\right)$ for $R \leq R_{n}$ and conclude that

$$
\mu(F) \leq \mu\left(B\left(\bar{x}, j_{n} R\right)\right) \leq c B(\bar{x}, R)
$$

for some constant $c$ depending on $n$ but not on $R$, that is (v). This also implies that $\mu(F)$ is comparable to $\mu\left(Q_{\beta}^{(n+1), k}\right)$ for any of the cubes defining $F$. Hence we can now prove that the doubling constant of $F$ only depends on $n$. Namely, revising the last part of the proof of Corollary 14 we can see that inequality

$$
c_{0} \mu\left(Q_{\alpha}^{k}\right) \geq c_{n, k} \mu\left(F_{k}\right)
$$

now rewrites as

$$
c_{0} \mu\left(Q_{\beta}^{(n+1), k}\right) \geq c_{n} \mu(F)
$$

and we are done. 


\section{Hölder continuous functions}

In several problems related to singular or fractional integrals we will need Hölder continuous cutoff functions adapted to concentric balls. This construction is classical and does not depend on the doubling condition, so can be performed in any $\Omega_{n}$ as in usual spaces of homogeneous type.

Fix $\Omega_{n}$. The function $\rho$ is a quasidistance in $\Omega_{n}$, hence by known results of Macias-Segovia 25. Thm. 2] we can build a new quasidistance $d$ in $\Omega_{n}$, equivalent to $\rho$ in $\Omega_{n}$, and such that for some $\alpha \in(0,1) d$ is of order $\alpha$, which means that

$$
\left|d\left(x_{1}, y\right)-d\left(x_{2}, y\right)\right| \leq c d\left(x_{1}, x_{2}\right)^{\alpha}\left\{d\left(x_{1}, y\right)^{1-\alpha}+d\left(x_{2}, y\right)^{1-\alpha}\right\}
$$

for some constant $c>0$, any $x, y, z \in \Omega_{n}$. Here and in the following, saying that two functions $\rho_{1}(x, y), \rho_{2}(x, y)$ are equivalent in $\Omega_{n}$ means that for two positive constants $c_{1}, c_{2}>0$ we have

$$
c_{1} \rho_{1}(x, y) \leq \rho_{2}(x, y) \leq c_{2} \rho_{1}(x, y) \text { for any } x, y \in \Omega_{n} .
$$

It is worthwhile to note that the exponent $\alpha$ in (45) depends on $n$; from the proof given in 25. Thm. 2] we read $\alpha=1 / \log _{2}\left(3 B_{n}^{2}\right)$, which is not optimal in the sense that for $B_{n}=1$ (that is when $\rho$ is a distance) does not say that (45) holds with $\alpha=1$.

Let us write $B_{d}(x, r)$ for the $d$-ball of center $x$ and radius $r$. Now, for any $x_{0} \in \Omega_{n}$ with $B_{d}\left(x_{0}, 2 r\right) \subset \Omega_{n}$ we can define the function

$$
\phi(x)=\psi\left(d\left(x, x_{0}\right)\right)
$$

where

$$
\psi(t)= \begin{cases}1 & 0 \leq t \leq r \\ 2-t / r & r \leq t \leq 2 r \\ 0 & t \geq 2 r\end{cases}
$$

A standard computation exploiting (45) and the equivalence between $\rho$ and $d$ allows to prove the following:

Proposition 17 For any $\Omega_{n}$ there exists an exponent $\alpha>0$ and two constants $c_{1}<1, c_{2}>2$, such that for any $x_{0} \in \Omega_{n}$ and $r>0$ with $B\left(x, c_{2} r\right) \subset \Omega_{n}$ there exists a function $\phi$ with the following properties:

$$
\begin{aligned}
0 & \leq \phi(x) \leq 1 \\
\phi(x) & =1 \text { for } x \in B\left(x_{0}, c_{1} r\right) \\
\phi(x) & =0 \text { for } x \notin B\left(x_{0}, c_{2} r\right) \\
\left|\phi\left(x_{1}\right)-\phi\left(x_{2}\right)\right| & \leq c\left(\frac{\rho\left(x_{1}, x_{2}\right)}{r}\right)^{\alpha} \text { for any } x_{1}, x_{2} \in \Omega_{n} .
\end{aligned}
$$


The cutoff function $\phi$ belongs to the space $C_{0}^{\alpha}\left(\Omega_{n}\right)$. Note that we can build such cutoff functions only for $\alpha \leq \alpha_{0}$ where the threshold $\alpha_{0}$ depends on the space $\Omega_{n}$. We will briefly write

$$
\phi \in C_{0}^{\alpha}\left(\Omega_{n}\right), B\left(x_{0}, c_{1} r\right) \prec \phi \prec B\left(x_{0}, c_{2} r\right)
$$

to say that $\phi$ has all the properties stated in the above proposition.

By our assumption of regularity of the measure $\mu$, the above result [25, Thm. 2 ] also implies, by a fairly standard argument, that for any bounded Borel set $E$ we can build a Hölder continuous function which approximates in $L^{p}$ norm, for any $p \in[1, \infty)$, the characteristic function of $E$. Therefore the following density result holds:

Proposition 18 For any $\Omega_{n}$ there exists $\alpha_{0}>0$, depending on $n$, such that for any $\alpha \in\left(0, \alpha_{0}\right]$, any $p \in[1, \infty)$, the space $C^{\alpha}\left(\Omega_{n}\right)$ is dense in $L^{p}\left(\Omega_{n}\right)$. If $\rho$ is a distance we can take $\alpha_{0}=1$.

We leave the details to the interested reader. Note that this is the only point of the theory where we use the assumption of regularity of $\mu$; moreover, this assumption could actually be removed. Namely, refining an argument contained in [20. Thm. 2.2.2], it is possible to prove that, under our assumptions (H1), (H2), (H4), any positive Borel measure on $\Omega$ has the regularity property which is used in the proof of this proposition.

\section{$5 \quad$ Local singular and fractional integrals}

We now want to develop a theory of singular and fractional integrals in locally homogeneous space. We are interested in situations, which typically occur when dealing with local a-priori estimates for subelliptic PDEs, where one builds singular kernels $K(x, y)$ which are naturally defined only locally, say for $x, y$ belonging to some ball $B\left(\bar{x}, R_{0}\right) \subset \Omega_{n+1}$ with $\bar{x} \in \Omega_{n}$. Starting from this kernel, one builds a new one of the kind

$$
\widetilde{K}(x, y)=a(x) K(x, y) b(y)
$$

where $a, b$ are suitable cutoff functions both supported in $B\left(\bar{x}, R_{0}\right)$. This $\widetilde{K}$ has the better property of being defined in the whole $\Omega_{n+1} \times \Omega_{n+1}$ (except the diagonal $x=y$ ); the integral operator with kernel $\widetilde{K}$ can be the right object to prove a local estimate, of $L^{p}$ or $C^{\alpha}$ type. We can use the Hölder continuous cutoff functions built in the previous section to define a kernel $\widetilde{K}$ supported in $B(\bar{x}, R) \times B(\bar{x}, R)$, and exploit the fact that $B(\bar{x}, R)$ is in turn essentially contained in a space of homogeneous type (see Theorem 15 ). Then, we would like to apply to the singular or fractional integral defined by $\widetilde{K}$ some existing results from the theory of spaces of homogeneous type. This requires checking that $\widetilde{K}$ satisfies globally, in the space of homogeneous type where we have embedded it, suitable properties: standard estimates, cancellation properties and so on. 
The following preliminary construction and results serve to this aim. Moreover, in view of the commutator theorems we are going to prove, we want to further shrink the support of $\widetilde{K}$, if necessary. This is the reason why we introduce a second variable radius $R<R_{0}$. We keep assuming that $\left(\Omega,\left\{\Omega_{n}\right\}_{n=1}^{\infty}, \rho, \mu\right)$ be a locally homogeneous space. Moreover, we make the following:

Assumption (H7). For fixed $\Omega_{n}, \Omega_{n+1}$, and a fixed ball $B\left(\bar{x}, R_{0}\right)$, with $\bar{x} \in \Omega_{n}$ and $R_{0}<2 \varepsilon_{n}$ (hence $B\left(\bar{x}, R_{0}\right) \subset \Omega_{n+1}$ ), let $K(x, y)$ be a measurable function defined for $x, y \in B\left(\bar{x}, R_{0}\right), x \neq y$. Let $R>0$ be any number satisfying

$$
c R \leq R_{0}
$$

for some $c>1$ which will be chosen in the proof of the next Proposition; let $a, b \in C_{0}^{\alpha}\left(\Omega_{n+1}\right), B\left(\bar{x}, c_{1} R\right) \prec a \prec B\left(\bar{x}, c_{2} R\right), B\left(\bar{x}, c_{3} R\right) \prec b \prec B\left(\bar{x}, c_{4} R\right)$ (see Proposition 17) for some fixed constants $c_{i} \in(0,1), i=1, \ldots, 4$. The new kernel

$$
\widetilde{K}(x, y)=a(x) K(x, y) b(y)
$$

can be considered defined in the whole $\Omega_{n+1} \times \Omega_{n+1} \backslash\{x=y\}$. Then:

Proposition 19 Under assumption ( $\mathrm{H}^{\text {Y }}$ ) we have:

(i) Assume $K$ satisfies for some $\nu \in[0,1)$ the following standard estimates:

$$
|K(x, y)| \leq \frac{A \rho(x, y)^{\nu}}{\mu(B(x, \rho(x, y)))}
$$

for $x, y \in B\left(\bar{x}, R_{0}\right), x \neq y$, and

$$
\left|K\left(x_{0}, y\right)-K(x, y)\right|+\left|K\left(y, x_{0}\right)-K(y, x)\right| \leq \frac{B \rho\left(x_{0}, y\right)^{\nu}}{\mu\left(B\left(x_{0}, \rho\left(x_{0}, y\right)\right)\right)}\left(\frac{\rho\left(x_{0}, x\right)}{\rho\left(x_{0}, y\right)}\right)^{\beta}
$$

for any $x_{0}, x, y \in B\left(\bar{x}, R_{0}\right)$ with $\rho\left(x_{0}, y\right)>M \rho\left(x_{0}, x\right)$, some $\beta>0, M>1$. $\left(M \geq 2 B_{n+1}\right.$, so that condition $\rho\left(x_{0}, y\right)>M \rho\left(x_{0}, x\right)$ implies the comparability of $\rho\left(x_{0}, y\right)$ and $\left.\rho(x, y)\right)$.

Then $\widetilde{K}$ satisfies the same bound (48) for any $x, y \in \Omega_{n+1}, x \neq y$ and a bound (49) (with a different constant $B^{\prime}$ ) for any $x_{0}, x, y \in \Omega_{n+1}$, with $\rho\left(x_{0}, y\right)>$ $\bar{M}\left(x_{0}, x\right)$, provided $\alpha \geq \beta$ (where $\alpha$ is the Hölder exponent related to the cutoff functions defining $\widetilde{K}$ ); the new constant $B^{\prime}$ depends on $A, B$ and $n$ (but not on $R)$.

(ii) Assume $K$ satisfies (48) with $\nu=0$ and the following cancellation property:

there exists $C>0$ such that for a.e. $x \in B\left(\bar{x}, R_{0}\right)$ and every $\varepsilon_{1}, \varepsilon_{2}$ such that $0<\varepsilon_{1}<\varepsilon_{2}$ and $B_{\rho^{\prime}}\left(x, \varepsilon_{2}\right) \subset \Omega_{n+1}$

$$
\left|\int_{\Omega_{n+1}, \varepsilon_{1}<\rho^{\prime}(x, y)<\varepsilon_{2}} K(x, y) d \mu(y)\right|+\left|\int_{\Omega_{n+1}, \varepsilon_{1}<\rho^{\prime}(x, z)<\varepsilon_{2}} K(z, x) d \mu(z)\right| \leq C,
$$


where $\rho^{\prime}$ is any quasidistance equivalent to $\rho$ in $\Omega_{n+1}$ and $B_{\rho^{\prime}}$ denotes $\rho^{\prime}$-balls.

Then $\widetilde{K}$ satisfies a similar cancellation property (with a different constant $\left.C^{\prime}\right)$ for a.e. $x \in \Omega_{n+1}, 0<\varepsilon_{1}<\varepsilon_{2}<\infty$. The new constant $C^{\prime}$ depends on $A, C$ and $n$ (but not on $R$ ).

The same is true if, in the condition (50), we replace the integration over $\Omega_{n+1}$ with the integration over any measurable set containing $B(\bar{x}, R)$.

(iii) Assume $K$ satisfies the bound (i) and the following convergence condition: for a.e. $x \in B\left(\bar{x}, R_{0}\right)$ such that $B_{\rho^{\prime}}(x, R) \subset \Omega_{n+1}$ there exists

$$
h_{R}(x) \equiv \lim _{\varepsilon \rightarrow 0} \int_{\Omega_{n+1}, \varepsilon<\rho^{\prime}(x, y)<R} K(x, y) d \mu(y),
$$

where $\rho^{\prime}$ is any quasidistance equivalent to $\rho$ in $\Omega_{n+1}$.

Then for a.e. $x \in \Omega_{n+1}$, there exists

$$
\widetilde{h}(x) \equiv \lim _{\varepsilon \rightarrow 0} \int_{\Omega_{n+1}, \rho^{\prime}(x, y)>\varepsilon} \widetilde{K}(x, y) d \mu(y) .
$$

Remark 20 The presence of a function $\rho^{\prime}$ possibly different from $\rho$ (but equivalent to it) in conditions (ii)-(iii) adds flexibility to the theory: it is sometimes easier to check these conditions for a $\rho^{\prime}$ different from $\rho$. For instance, when dealing with local estimates for operators structured on Hörmander's vector fields, typically $\rho$ will be the Carnot-Carathéodory distance induced by the vector fields, while $\rho^{\prime}$ will be the quasidistance defined by Rothschild-Stein in [27].

Proof. The first part of (i) is obvious. To prove the second part, let us write, for $x_{0}, x, y \in \Omega_{n+1}, \rho\left(x_{0}, y\right)>M \rho\left(x_{0}, x\right)$ :

$$
\begin{aligned}
\left|\widetilde{K}\left(x_{0}, y\right)-\widetilde{K}(x, y)\right| & \leq\left|a\left(x_{0}\right) K\left(x_{0}, y\right) b(y)-a\left(x_{0}\right) K(x, y) b(y)\right| \\
& +\left|a\left(x_{0}\right) K(x, y) b(y)-a(x) K(x, y) b(y)\right| \\
& =I+I I .
\end{aligned}
$$

By (49),

$$
I \leq\left|a\left(x_{0}\right) b(y)\right| \frac{B \rho\left(x_{0}, y\right)^{\nu}}{\mu\left(B\left(x_{0}, \rho\left(x_{0}, y\right)\right)\right)}\left(\frac{\rho\left(x_{0}, x\right)}{\rho\left(x_{0}, y\right)}\right)^{\beta}
$$

when $x_{0}, x, y \in B\left(\bar{x}, R_{0}\right)$. Since the quantity $\left|a\left(x_{0}\right) b(y)\right|$ does not vanish only if $x_{0}, y \in B(\bar{x}, R)$, it is enough to consider what happens when $x_{0}, y \in B(\bar{x}, R)$ and $x \notin B(\bar{x}, R)$. We have

$$
\begin{aligned}
\rho(x, \bar{x}) & \leq B_{n+1}\left(\rho\left(x, x_{0}\right)+\rho\left(x_{0}, \bar{x}\right)\right) \leq B_{n+1}\left(\frac{1}{M} \rho\left(x_{0}, y\right)+R\right) \\
& \leq B_{n+1}\left(\frac{1}{M} B_{n+1}\left(\rho\left(x_{0}, \bar{x}\right)+\rho(\bar{x}, y)\right)+R\right) \\
& \leq B_{n+1}\left(\frac{1}{M} B_{n+1} 2 R+R\right) \leq 2 B_{n+1} R
\end{aligned}
$$


(by our assumption $M \geq 2 B_{n+1}$ ). Hence, if in Assumption (H7) we take

$$
c R \leq R_{0} \text { with } c>2 B_{n+1},
$$

we have $\rho(x, \bar{x}) \leq R_{0}$, and (51) holds for any $x_{0}, x, y \in \Omega_{n+1}$ with $\rho\left(x_{0}, y\right)>$ $M \rho\left(x_{0}, x\right)$.

Now,

$$
I I=\left|a\left(x_{0}\right)-a(x)\right||K(x, y) b(y)|
$$

by Proposition 17 and (48), for $x, y \in B\left(\bar{x}, R_{0}\right)$,

$$
\begin{aligned}
& \leq c\left(\frac{\rho\left(x_{0}, x\right)}{R}\right)^{\alpha}|K(x, y) b(y)| \\
& \leq c \frac{B \rho\left(x_{0}, y\right)^{\nu}}{\mu\left(B\left(x_{0}, \rho\left(x_{0}, y\right)\right)\right)}\left(\frac{\rho\left(x_{0}, x\right)}{R}\right)^{\alpha}
\end{aligned}
$$

since $\rho\left(x_{0}, y\right)$ is comparable to $\rho(x, y)$.

The term $I I$ does not vanish only if $y \in B(\bar{x}, R)$ and $x$ or $x_{0}$ belongs to $B(\bar{x}, R)$.

If $y, x_{0} \in B(\bar{x}, R)$ then $\rho\left(x_{0}, y\right) \leq 2 B_{n+1} R$. On the other hand, condition $\rho\left(x_{0}, y\right)>M \rho\left(x_{0}, x\right)$ with $M \geq 2 B_{n+1}$ implies

$$
\rho\left(x_{0}, y\right) \leq 2 B_{n+1} \rho(x, y) .
$$

Hence if $y, x \in B(\bar{x}, R)$ then $\rho(x, y) \leq 2 B_{n+1} R$ and $\rho\left(x_{0}, y\right) \leq\left(2 B_{n+1}\right)^{2} R$. So, in any case $\rho\left(x_{0}, y\right) \leq c_{1} R$, and (53) gives

$$
\begin{aligned}
I I & \leq c \frac{B \rho(x, y)^{\nu}}{\mu\left(B\left(x_{0}, \rho(x, y)\right)\right)}\left(\frac{\rho\left(x_{0}, x\right)}{\rho\left(x_{0}, y\right)}\right)^{\alpha} \\
& \leq c \frac{B \rho\left(x_{0}, y\right)^{\nu}}{\mu\left(B\left(x_{0}, \rho\left(x_{0}, y\right)\right)\right)}\left(\frac{\rho\left(x_{0}, x\right)}{\rho\left(x_{0}, y\right)}\right)^{\beta}
\end{aligned}
$$

for any $\beta \leq \alpha, x, y \in B\left(\bar{x}, R_{0}\right)$. It is now enough to check what happens for $y, x_{0} \in B(\bar{x}, R)$ and $x \notin B\left(\bar{x}, R_{0}\right)$. Reasoning like above, the conditions $y, x_{0} \in$ $B(\bar{x}, R)$ imply $\rho(x, \bar{x}) \leq 2 B_{n+1} R<R_{0}$ by (52), hence $x \notin B\left(\bar{x}, R_{0}\right)$ simply cannot happen.

To prove (ii), let $x \in \Omega_{n+1}$ and consider, for any $\varepsilon_{2}>\varepsilon_{1}>0$,

$$
A \equiv \int_{\Omega_{n+1}, \varepsilon_{1}<\rho^{\prime}(x, y)<\varepsilon_{2}} \widetilde{K}(x, y) d \mu(y)=a(x) \int_{\Omega_{n+1}, \varepsilon_{1}<\rho^{\prime}(x, y)<\varepsilon_{2}} K(x, y) b(y) d \mu(y) .
$$

This quantity does not vanish only if $x \in B(\bar{x}, R)$; since also $b(y)$ does not vanish only for $y \in B(\bar{x}, R)$, the integrand does not vanish only if $\rho^{\prime}(x, y) \leq c^{\prime} R$ for some $c^{\prime}$. Choose the number $c$ in (46) so that $c R \leq R_{0}$ implies $c^{\prime} R \leq R_{0}$ 
and $B\left(x, c^{\prime} R\right) \subset \Omega_{n+1}$. Then

$$
\begin{aligned}
A & =a(x) \int_{\Omega_{n+1}, \varepsilon_{1}<\rho^{\prime}(x, y)<\min \left(\varepsilon_{2}, c^{\prime} R\right)} K(x, y) b(y) d \mu(y) \\
& =a(x) b(x) \int_{\Omega_{n+1}, \varepsilon_{1}<\rho^{\prime}(x, y)<\min \left(\varepsilon_{2}, c^{\prime} R\right)} K(x, y) d \mu(y)+ \\
& +\int_{\Omega_{n+1}, \varepsilon_{1}<\rho^{\prime}(x, y)<\min \left(\varepsilon_{2}, c^{\prime} R\right)} K(x, y)[b(y)-b(x)] d \mu(y) \equiv A_{1}+A_{2} .
\end{aligned}
$$

Now by (50) we can bound

$$
\left|A_{1}\right| \leq C|a(x) b(x)| \leq C
$$

while

$$
\begin{aligned}
\left|A_{2}\right| & \leq \int_{\Omega_{n+1}, \varepsilon_{1}<\rho^{\prime}(x, y)<\min \left(\varepsilon_{2}, c^{\prime} R\right)}|K(x, y)| c\left(\frac{\rho(x, y)}{R}\right)^{\alpha} d \mu(y) \\
& \leq \frac{c}{R^{\alpha}} \int_{\Omega, \rho(x, y)<c^{\prime \prime} R} \frac{A \rho(x, y)^{\alpha}}{\mu(B(x, \rho(x, y)))} d \mu(y) \leq \frac{c}{R^{\alpha}} c^{\prime \prime \prime} R^{\alpha} \equiv C^{\prime} .
\end{aligned}
$$

In the last inequality we have applied a standard estimate in spaces of homogeneous type (since the integral is extended to a ball centered at a point of $\Omega_{n+1}$ and contained in $\Omega_{n+2}$ we can apply the local doubling condition):

$$
\begin{aligned}
& \int_{\Omega, \rho(x, y)<c^{\prime \prime} R} \frac{\rho(x, y)^{\alpha}}{\mu(B(x, \rho(x, y)))} d \mu(y) \\
& \leq \sum_{k=0}^{\infty} \int_{\Omega_{n+1}, \frac{c^{\prime \prime} R}{2^{k+1}} \leq \rho(x, y)<\frac{c^{\prime \prime} R}{2^{k}}} \frac{\rho(x, y)^{\alpha}}{\mu(B(x, \rho(x, y)))} d \mu(y) \\
& \leq \sum_{k=0}^{\infty}\left(\frac{c^{\prime \prime} R}{2^{k}}\right)^{\alpha} \frac{\mu\left(B\left(x, \frac{c^{\prime \prime} R}{2^{k}}\right)\right)}{\mu\left(B\left(x, \frac{c^{\prime \prime} R}{2^{k+1}}\right)\right)} \leq \sum_{k=0}^{\infty} C_{n+1}\left(\frac{c^{\prime \prime} R}{2^{k}}\right)^{\alpha}=c^{\prime \prime \prime} R^{\alpha} .
\end{aligned}
$$

To prove (iii), let us consider, for $x \in \Omega_{n+1}$ and $0<\varepsilon_{1}<\varepsilon_{2}$,

$$
\begin{aligned}
& \int_{\Omega_{n+1}, \rho^{\prime}(x, y)>\varepsilon_{2}} \widetilde{K}(x, y) d \mu(y)-\int_{\Omega_{n+1}, \rho^{\prime}(x, y)>\varepsilon_{1}} \widetilde{K}(x, y) d \mu(y) \\
& =a(x) \int_{\Omega_{n+1}, \varepsilon_{1}<\rho^{\prime}(x, y) \leq \varepsilon_{2}} K(x, y) b(y) d \mu(y) \equiv A\left(\varepsilon_{1}, \varepsilon_{2}, x\right) .
\end{aligned}
$$

The quantity $A\left(\varepsilon_{1}, \varepsilon_{2}, x\right)$ does not vanish only if $x \in B(\bar{x}, R)$; for this $x$ and $R$ small enough we have $B(x, R) \subset \Omega_{n+1}$, hence we can write

$$
\begin{aligned}
A\left(\varepsilon_{1}, \varepsilon_{2}, x\right) & =a(x) b(x) \int_{\Omega_{n+1}, \varepsilon_{1}<\rho^{\prime}(x, y) \leq \varepsilon_{2}} K(x, y) d \mu(y) \\
& +a(x) \int_{\Omega_{n+1}, \varepsilon_{1}<\rho^{\prime}(x, y) \leq \varepsilon_{2}} K(x, y)[b(y)-b(x)] d \mu(y) \\
& \equiv A_{1}\left(\varepsilon_{1}, \varepsilon_{2}, x\right)+A_{2}\left(\varepsilon_{1}, \varepsilon_{2}, x\right)
\end{aligned}
$$


and, by our assumption on $K$,

$$
\lim _{\varepsilon_{1}, \varepsilon_{2} \rightarrow 0} A_{1}\left(\varepsilon_{1}, \varepsilon_{2}, x\right)=0
$$

On the other hand, reasoning as above, we get

$$
\left|A_{2}\left(\varepsilon_{1}, \varepsilon_{2}, x\right)\right| \leq \frac{c}{R^{\alpha}} \int_{\Omega, \rho(x, y)<c^{\prime \prime} \varepsilon_{2}} \frac{A \rho(x, y)^{\alpha}}{\mu(B(x, \rho(x, y)))} d \mu(y) \leq c\left(\frac{\varepsilon_{2}}{R}\right)^{\alpha}
$$

which also vanishes for $\varepsilon_{2} \rightarrow 0$. So the desired limit exists.

Theorem 21 ( $L^{p}$ and $C^{\eta}$ estimates for singular integrals) Let $K, \widetilde{K}$ be as in Assumption ( $\left.\mathrm{H}^{7}\right)$, with $K$ satisfying the standard estimates (i) with $\nu=0$, the cancellation property (ii) and the convergence condition (iii) stated in Proposition 19. If

$$
T f(x)=\lim _{\varepsilon \rightarrow 0} \int_{B(\bar{x}, R), \rho^{\prime}(x, y)>\varepsilon} \widetilde{K}(x, y) f(y) d \mu(y),
$$

then for any $p \in(1, \infty)$

$$
\|T f\|_{L^{p}(B(\bar{x}, R))} \leq c\|f\|_{L^{p}(B(\bar{x}, R))} .
$$

The constant $c$ depends on $p, n$ and the constants of $K$ involved in the assumptions (but not on $R$ ).

Moreover, $T$ satisfies a weak 1-1 estimate:

$$
\mu(\{x \in B(\bar{x}, R):|T f(x)|>t\}) \leq \frac{c}{t}\|f\|_{L^{1}(B(\bar{x}, R))} \text { for any } t>0 .
$$

Assume that, in addition, the kernel $K$ satisfies the condition

$$
\widetilde{h}(x) \equiv \lim _{\varepsilon \rightarrow 0} \int_{\rho^{\prime}(x, y)>\varepsilon} \widetilde{K}(x, y) d \mu(y) \in C^{\gamma}\left(\Omega_{n+1}\right)
$$

for some $\gamma>0$ (where $\rho^{\prime}$ is the same appearing in the assumed convergence condition (iii)). Then

$$
\|T f\|_{C^{\eta}(B(\bar{x}, R))} \leq c\|f\|_{C^{\eta}(B(\bar{x}, H R))}
$$

for any positive $\eta<\min (\alpha, \beta, \gamma)$ and some constant $H>1$ independent of $R$. (Recall that $\alpha$ is the Hölder exponent related to the cutoff functions defining $\widetilde{K}, \beta$ appears in the standard estimates (i) and $\gamma$ is the number in (54)).

The constant $c$ depends on $\eta, n, R$, the constants involved in the assumptions on $K$, and the $C^{\gamma}$ norm of $\widetilde{h}$.

Proof. By Theorem 15 there exists a space of homogeneous type $F$ such that

$$
B(\bar{x}, R) \backslash E \subset F \subset \Omega_{n+1}
$$


and the doubling constant of $F$ only depends on $n$. By our assumptions on $K$ and Proposition 19, the operator $T$ satisfies all the assumptions of Theorem 41 (about singular integrals in spaces of homogeneous type), so that

$$
\|T f\|_{L^{p}(B(\bar{x}, R))} \leq\|T f\|_{L^{p}(F)} \leq c\|f\|_{L^{p}(F)}
$$

with $c$ depending on $p, n$ and the constants involved in the assumptions about $K$. Applying the inequality to $f \in L^{p}(B(\bar{x}, R)$ ) (having set $f=0$ outside this ball), we get

$$
\|T f\|_{L^{p}(B(\bar{x}, R))} \leq c\|f\|_{L^{p}(B(\bar{x}, R))} .
$$

The same argument gives the weak 1-1 estimate for $T$.

For the $C^{\eta}$ case a similar argument applies; we now apply Theorem 42 and get

$$
\|T f\|_{C^{\eta}(F)} \leq c\|f\|_{C^{\eta}(F)}
$$

with $c$ depending on $\eta, n$, the constants involved in the assumptions about $K$, the $C^{\gamma}$ norm of $h$, and also $\operatorname{diam} F$, that is $R$. Moreover, $B(\bar{x}, R) \subset \bar{F}$ hence

$$
\|T f\|_{C^{\eta}(B(\bar{x}, R))} \leq\|T f\|_{C^{\eta}(\bar{F})}=\|T f\|_{C^{\eta}(F)} \leq c\|f\|_{C^{\eta}(F)} .
$$

A difference with the $L^{p}$ case is that now we cannot set $f=0$ outside the ball $B(\bar{x}, R)$ preserving its Hölder continuity, therefore we can just write

$$
\|T f\|_{C^{\eta}(B(\bar{x}, R))} \leq c\|f\|_{C^{\eta}(B(\bar{x}, H R))}
$$

since, for some $H>1$ independent of $R$, we have $F \subset B(\bar{x}, H R)$, as seen in the proof of Theorem 15 .

Remark 22 (Estimates for $C_{0}^{\eta}$ functions) In the applications of this theory to local a priori estimates for PDEs, the function $f$ is usually compactly supported, so that we can apply (55) to $f \in C_{0}^{\eta}(B(\bar{x}, R))$, getting the more appealing inequality

$$
\|T f\|_{C^{\eta}(B(\bar{x}, R))} \leq c\|f\|_{C^{\eta}(B(\bar{x}, R))} .
$$

Moreover, applying this inequality to functions $f \in C_{0}^{\eta}(B(\bar{x}, r))$ with $r<R$ we can get a a bound

$$
\|T f\|_{C^{\eta}(B(\bar{x}, r))} \leq c\|f\|_{C^{\eta}(B(\bar{x}, r))}
$$

with $c$ depending on $R$ but not on $r$.

Remark 23 (Checking assumption (54)) Assumption (54) can be the most troublesome to check in concrete applications (apart from classical cases in which $\widetilde{h}$ is zero, or is constant). In some applications to subelliptic equations, the kernel $\widetilde{K}(x, y)$ happens to be a perturbation of a simpler kernel which has vanishing integral over spherical shells; in these cases, one can prove that the limit

$$
\lim _{\varepsilon \rightarrow 0} \int_{\rho^{\prime}(x, y)>\varepsilon} \widetilde{K}(x, y) d \mu(y)
$$


equals to the integral of a nonsingular kernel satisfying standard estimates 48)(49) for some $\nu>0$. It is then helpful to recall that such an integral always belongs to a Hölder space, as will follow from Theorem 25, since it can be regarded as $T(1)$, where the constant 1 is Hölder continuous and $T$ is a fractional integral..

Theorem 24 ( $L^{p}-L^{q}$ estimate for fractional integrals) Let $K, \widetilde{K}$ be as in Assumption ( $\left.\mathrm{H}^{7}\right)$, with $K$ satisfying the growth condition

$$
0 \leq K(x, y) \leq \frac{c}{\mu(B(x, \rho(x, y)))^{1-\nu}}
$$

for some $\nu \in(0,1), c>0$, any $x, y \in B\left(\bar{x}, R_{0}\right), x \neq y$. If

$$
I_{\nu} f(x)=\int_{B(\bar{x}, R)} \widetilde{K}(x, y) f(y) d \mu(y)
$$

then, for any $p \in\left(1, \frac{1}{\nu}\right), \frac{1}{q}=\frac{1}{p}-\nu$ there exists $c$ such that

$$
\left\|I_{\nu} f\right\|_{L^{q}(B(\bar{x}, R))} \leq c\|f\|_{L^{p}(B(\bar{x}, R))}
$$

for any $f \in L^{p}(B(\bar{x}, R))$. The constant $c$ depends on $p, n$, and the constants of $K$ involved in the assumptions (but not on $R$ ).

Proof. This theorem follows from the analog result which holds in spaces of homogeneous type, that is Theorem 43 , by a similar argument to that used in the proof of Theorem 21

The analog $C^{\eta}$ estimate for fractional integrals is better stated under slightly different assumptions on the kernel. In the applications of the theory that we have in mind, where the measure of a ball is equivalent to a fixed power of the radius, both the theorems will be applicable.

Theorem 25 ( $C^{\eta}$ estimate for fractional integrals) Let $K, \widetilde{K}$ be as in $A s$ sumption ( $\left.\mathrm{H}^{\gamma}\right)$, with $K$ satisfying (48) and (49) for some $\nu \in(0,1), \beta>0$. If

$$
I_{\nu} f(x)=\int_{B(\bar{x}, R)} \widetilde{K}(x, y) f(y) d \mu(y),
$$

then, for any $\eta<\min (\alpha, \beta, \nu)$

$$
\left\|I_{\nu} f\right\|_{C^{\eta}(B(\bar{x}, R))} \leq c\|f\|_{C^{\eta}(B(\bar{x}, H R))} .
$$

The constant $c$ depends on $\eta, n, R$ and the constants of $K$ involved in the assumptions; the number $H$ only depends on $n$.

Reasoning as in Remark22, we can also say that for functions $f \in C_{0}^{\eta}(B(\bar{x}, r))$ with $r<R$ the following bound holds

$$
\left\|I_{\nu} f\right\|_{C^{\eta}(B(\bar{x}, r))} \leq c\|f\|_{C^{\eta}(B(\bar{x}, r))}
$$

with $c$ depending on $R$ but not on $r$.

Proof. This theorem follows from Proposition 19] and the analog result which holds in spaces of homogeneous type, that is Theorem 44, by an argument similar to that used in the proof of the $C^{\eta}$ case in Theorem 21. 


\section{Local and global $B M O$ and $V M O$ spaces}

Let $\left(\Omega,\left\{\Omega_{n}\right\}_{n=1}^{\infty}, \rho, \mu\right)$ be a locally homogeneous space.

Definition 26 (Local $B M O$ and $V M O$ spaces) For any function $u \in L^{1}\left(\Omega_{n+1}\right)$, and $r>0$, with $r \leq \varepsilon_{n}$, set

$$
\eta_{u, \Omega_{n}, \Omega_{n+1}}^{*}(r)=\sup _{t \leq r} \sup _{x_{0} \in \Omega_{n}} \frac{1}{\mu\left(B\left(x_{0}, t\right)\right)} \int_{B\left(x_{0}, t\right)}\left|u(x)-u_{B}\right| d \mu(x),
$$

where $u_{B}=\mu\left(B\left(x_{0}, t\right)\right)^{-1} \int_{B\left(x_{0}, t\right)} u$. We say that $u \in B M O_{l o c}\left(\Omega_{n}, \Omega_{n+1}\right)$ if

$$
\|u\|_{B M O_{l o c}\left(\Omega_{n}, \Omega_{n+1}\right)}=\sup _{r \leq \varepsilon_{n}} \eta_{u, \Omega_{n}, \Omega_{n+1}}^{*}(r)<\infty .
$$

We say that $u \in V M O_{\text {loc }}\left(\Omega_{n}, \Omega_{n+1}\right)$ if $u \in B M O_{l o c}\left(\Omega_{n}, \Omega_{n+1}\right)$ and

$$
\eta_{u, \Omega_{n}, \Omega_{n+1}}^{*}(r) \rightarrow 0 \text { as } r \rightarrow 0 \text {. }
$$

The function $\eta_{u, \Omega_{n}, \Omega_{n+1}}^{*}$ will be called VMO local modulus of $u$ in $\left(\Omega_{n}, \Omega_{n+1}\right)$.

Note that in the previous definition we integrate $u$ over balls centered at points of $\Omega_{n}$ and enclosed in $\Omega_{n+1}$. This is a fairly natural definition if we want to avoid integrating over the intersection $B\left(x_{0}, t\right) \cap \Omega_{n}$. We will need also the following standard

Definition 27 ( $B M O$ and $V M O$ spaces over a homogeneous space) Let $S$ be a subset of $\Omega$ which is a space of homogeneous type ( $S$ can be a single cube $Q_{\alpha}^{k}$, or the set $F$ built in Theorem 15, or the whole $\left.\bigcup_{\alpha \in I_{k}} Q_{\alpha}^{k}\right)$. For any function $u \in L^{1}(S)$ and $r>0$, set

$$
\eta_{u, S}(r)=\sup _{t \leq r} \sup _{x_{0} \in S} \frac{1}{\mu\left(B\left(x_{0}, t\right) \cap S\right)} \int_{B\left(x_{0}, t\right) \cap S}\left|u(x)-u_{B \cap S}\right| d \mu(x),
$$

where $u_{B \cap S}=\mu\left(B\left(x_{0}, t\right) \cap S\right)^{-1} \int_{B\left(x_{0}, t\right) \cap S} u$. We say that $u \in B M O(S)$ if

$$
\|u\|_{B M O(S)}=\sup _{r>0} \eta_{u, S}(r)<\infty .
$$

We say that $u \in V M O(S)$ if $u \in B M O(S)$ and $\eta_{u, S}(r) \rightarrow 0$ as $r \rightarrow 0$. The function $\eta_{u, S}$ will be called VMO modulus of $u$ in $S$.

The useful link between the two notions of $B M O$ is contained in the next Proposition. Here we have to consider families of dyadic cubes adapted to different sets $\Omega_{n}$, hence we need to add an extra index to our symbols.

Proposition 28 For fixed $n$ and $\bar{x} \in \Omega_{n}$, let $B(\bar{x}, R), F$ be as in Theorem 15 (recall that $\left.F \subset \Omega_{n+2}\right)$. Let $f \in B M O_{l o c}\left(\Omega_{n+2}, \Omega_{n+3}\right)$, then

$$
\|f\|_{B M O(F)} \leq c \eta_{f, \Omega_{n+2}, \Omega_{n+3}}^{*}(c R) \leq c\|f\|_{B M O_{l o c}\left(\Omega_{n+2}, \Omega_{n+3}\right)}
$$

for a constant $c$ depending on $n$ but independent of $R$. In particular, given $f \in V M O_{l o c}\left(\Omega_{n+2}, \Omega_{n+3}\right)$, the norm $\|f\|_{B M O(F)}$ can be taken as small as we want, for fixed $n$ and $R$ small enough. 
Proof. The second inequality holds by definition, so let us prove the first. With the notation used in the proof of Theorem 15, let $x \in F$, that is $x \in Q_{\beta}^{(n+1), k}$ for some $Q_{\beta}^{(n+1), k}$ intersecting $B\left(z_{\alpha}^{(n), k}, h_{n} \delta_{(n)}^{k}\right)$. In particular, $x \in \Omega_{n+2}$. Recall that $\delta_{(n)}^{k+1}<R \leq \delta_{(n)}^{k}$. We want to bound, for any $r>0$,

$$
I \equiv \frac{1}{\mu(B(x, r) \cap F)} \int_{B(x, r) \cap F}|f(y)-c| d \mu(y)
$$

with $c$ to be chosen later. Let us distinguish the cases:

(i) $r \leq \delta_{(n+1)}^{k}$. Then

$$
\mu(B(x, r) \cap F) \geq \mu\left(B(x, r) \cap Q_{\beta}^{(n+1), k}\right) \geq c_{0,(n+1)} \mu(B(x, r))
$$

(see (12)), hence choosing $c=f_{B(x, r)}$

$$
\begin{aligned}
I & \leq \frac{c}{\mu(B(x, r))} \int_{B(x, r)}\left|f(y)-f_{B(x, r)}\right| d \mu(y) \\
& \leq c \eta_{f, \Omega_{n+2}, \Omega_{n+3}}^{*}\left(\delta_{(n+1)}^{k}\right) \leq c \eta_{f, \Omega_{n+2}, \Omega_{n+3}}^{*}\left(c_{n} R\right)
\end{aligned}
$$

since $\delta_{(n+1)} \leq \delta_{(n)}$ and $\delta_{(n)}^{k+1}<R$.

(ii) $r>\delta_{(n+1)}^{k}$. Then

$$
\mu(B(x, r) \cap F) \geq \mu\left(B(x, r) \cap Q_{\beta}^{(n+1), k}\right) \geq c_{0,(n+1)} \mu\left(Q_{\beta}^{(n+1), k}\right)
$$

(see (12)), which in turn is equivalent to $\mu(F)$ because the two sets have comparable diameters and the first is contained in the second. Here we can apply the local doubling condition for balls of radius $\simeq \delta_{(n+1)}^{k}$ centered in $\Omega_{n+1}$ and contained in $\Omega_{n+2}$. Hence

$$
I \leq \frac{c}{\mu(F)} \int_{F}|f(y)-c| d \mu(y) .
$$

In turn (see the last part of the proof of Theorem 15), $F \subset B\left(\bar{x}, j_{n} R\right)$ with $\mu(F)$ comparable to $\mu\left(B\left(\bar{x}, j_{n} R\right)\right)$, therefore choosing $c=f_{B\left(\bar{x}, j_{n} R\right)}$ we get

$$
I \leq c \eta_{f, \Omega_{n+2}, \Omega_{n+3}}^{*}\left(c_{n} R\right)
$$

and we are done.

\section{Commutators of local singular and fractional integrals with BMO functions}

Theorem 29 (Commutators of local singular integrals) Let $K, \widetilde{K}$ be as in Assumption ( $\left.H^{7}\right)$, with $K$ satisfying the standard estimates (i) with $\nu=0$, 
the cancellation property (ii) and the convergence condition (iii) (see Proposition 19). If

$$
T f(x)=\lim _{\varepsilon \rightarrow 0} \int_{B(\bar{x}, R), \rho^{\prime}(x, y)>\varepsilon} \widetilde{K}(x, y) f(y) d \mu(y)
$$

and, for $a \in B M O_{l o c}\left(\Omega_{n+2}, \Omega_{n+3}\right)$, we set

$$
C_{a} f(x)=T(a f)(x)-a(x) T f(x),
$$

then for any $p \in(1, \infty)$ there exists $c>0$ such that

$$
\left\|C_{a} f\right\|_{L^{p}(B(\bar{x}, R))} \leq c\|a\|_{B M O_{l o c}\left(\Omega_{n+2}, \Omega_{n+3}\right)}\|f\|_{L^{p}(B(\bar{x}, R))} .
$$

Moreover, if $a \in V M O_{l o c}\left(\Omega_{n+2}, \Omega_{n+3}\right)$ for any $\varepsilon>0$ there exists $r>0$ such that for any $f \in L^{p}(B(\bar{x}, r))$ we have

$$
\left\|C_{a} f\right\|_{L^{p}(B(\bar{x}, r))} \leq \varepsilon\|f\|_{L^{p}(B(\bar{x}, r))} .
$$

The constant $c$ depends on $p, n$ and the constants of $K$ involved in the assumptions (but not on $R$ ); the constant $r$ also depends on the $V M O_{l o c}\left(\Omega_{n+2}, \Omega_{n+3}\right)$ modulus of $a$.

Proof. Proceeding like in the proof of Theorem 21, and with the same meaning of the symbols, we prove, applying Theorems 45 and 41 which hold in spaces of homogeneous type, that

$$
\left\|C_{a} f\right\|_{L^{p}(F)} \leq c\|a\|_{B M O(F)}\|f\|_{L^{p}(F)}
$$

for some constant $c$ depending on $p, n$ and the constants involved in the assumptions on $K$ (but not on $R$ ); in turn, by Proposition 28 the last quantity is bounded by

$$
c\|a\|_{B M O_{l o c}\left(\Omega_{n+2}, \Omega_{n+3}\right)}\|f\|_{L^{p}(F)} .
$$

Reasoning on the support of $f$ we get, like in the proof of Theorem 21,

$$
\left\|C_{a} f\right\|_{L^{p}(B(\bar{x}, R))} \leq c\|a\|_{B M O_{l o c}\left(\Omega_{n+2}, \Omega_{n+3}\right)}\|f\|_{L^{p}(B(\bar{x}, R))} .
$$

To prove the second assertion, we now observe that if we apply the $L^{p}$ continuity estimate

$$
\|T f\|_{L^{p}(B(\bar{x}, R))} \leq c\|f\|_{L^{p}(B(\bar{x}, R))}
$$

to functions $f \in L^{p}(B(\bar{x}, r))$ for any $r<R$, we find

$$
\|T f\|_{L^{p}(B(\bar{x}, r))} \leq c\|f\|_{L^{p}(B(\bar{x}, r))}
$$

so that the same operator $T$ is continuous on $L^{p}(B(\bar{x}, r))$, for any $r<R$, with a constant independent of $r$. (Recall that the number $R$ is involved in the definition of $T$ (through the cutoff functions), so that (59) is not the same as "(58) for $R$ small"). 
Take $r$ so small that for $x, y \in B(\bar{x}, r)$ we have $\widetilde{K}(x, y)=K(x, y)$. Then the kernel $\widetilde{K}$ satisfies in $B(\bar{x}, r)$ assumptions (i) in Proposition 19, with constants independent of $r$. We can therefore apply again the commutator theorem on spaces of homogeneous type (Theorem 45) to the operator $T$ on the space $F^{\prime}$ built as in Theorem 15 with $F^{\prime}$ essentially containing $B(\bar{x}, r)$ and and comparable with it, concluding that, for any $f \in L^{p}(B(\bar{x}, r))$,

$$
\begin{aligned}
\left\|C_{a} f\right\|_{L^{p}(B(\bar{x}, r))} & \leq\left\|C_{a} f\right\|_{L^{p}\left(F^{\prime}\right)} \leq c\|a\|_{B M O\left(F^{\prime}\right)}\|f\|_{L^{p}\left(F^{\prime}\right)} \\
& \leq c \eta_{a, \Omega_{n+2}, \Omega_{n+3}}^{*}\left(c_{n} r\right)\|f\|_{L^{p}(B(\bar{x}, r))}
\end{aligned}
$$

where we have applied again Proposition 28. Since in the last inequality the constants $c, c_{n}$ are independent of $r$, if $a \in V M O_{l o c}\left(\Omega_{n+2}, \Omega_{n+3}\right)$, for any $\varepsilon>0$ we can find $r$ small enough so that $c \eta_{a, \Omega_{n+2}, \Omega_{n+3}}^{*}\left(c_{1} r\right)<\varepsilon$, and we are done.

Theorem 30 (Positive commutators of local fractional integrals) Let $K, \widetilde{K}$ be as in Assumption ( $\mathrm{H}^{7}$ ), with $K$ satisfying the growth condition (56) for some $\nu>0$. If

$$
I_{\nu} f(x)=\int_{B(\bar{x}, R)} \widetilde{K}(x, y) f(y) d \mu(y)
$$

and, for $a \in B M O_{l o c}\left(\Omega_{n+2}, \Omega_{n+3}\right)$, we set

$$
C_{\nu, a} f(x)=\int_{B(\bar{x}, R)} \widetilde{K}(x, y)|a(x)-a(y)| f(y) d \mu(y)
$$

then, for any $p \in\left(1, \frac{1}{\nu}\right), \frac{1}{q}=\frac{1}{p}-\nu$ there exists $c$ such that

$$
\left\|C_{\nu, a} f\right\|_{L^{q}(B(\bar{x}, R))} \leq c\|a\|_{B M O_{l o c}\left(\Omega_{n+2}, \Omega_{n+3}\right)}\|f\|_{L^{p}(B(\bar{x}, R))}
$$

for any $f \in L^{p}(B(\bar{x}, R))$.

Moreover, if $a \in V M O_{l o c}\left(\Omega_{n+2}, \Omega_{n+3}\right)$ for any $\varepsilon>0$ there exists $r>0$ such that for any $f \in L^{p}(B(\bar{x}, r))$ we have

$$
\left\|C_{\nu, a} f\right\|_{L^{q}(B(\bar{x}, r))} \leq \varepsilon\|f\|_{L^{p}(B(\bar{x}, r))} .
$$

The constant $c$ depends on $p, \nu, n$ and the constants involved in the assumptions on $K$ (but not on $R$ ); the constant $r$ also depends on the $V M O_{l o c}\left(\Omega_{n+2}, \Omega_{n+3}\right)$ modulus of a.

Proof. Proceeding like in the proof of Theorem 29, and with the same meaning of the symbols, applying Theorem 46 which holds in spaces of homogeneous type, we get that

$$
\begin{aligned}
\left\|C_{\nu, a} f\right\|_{L^{q}(F)} & \leq c\|a\|_{B M O(F)}\|f\|_{L^{p}(F)} \\
& \leq c\|a\|_{B M O_{l o c}\left(\Omega_{n+2}, \Omega_{n+3}\right)}\|f\|_{L^{p}(F)}
\end{aligned}
$$

for any $p \in\left(1, \frac{1}{\nu}\right), \frac{1}{q}=\frac{1}{p}-\nu$. Like in the proof of Theorem 29, we have

$$
\left\|C_{\nu, a} f\right\|_{L^{q}(B(\bar{x}, R))} \leq c\|a\|_{B M O_{l o c}\left(\Omega_{n+2}, \Omega_{n+3}\right)}\|f\|_{L^{p}(B(\bar{x}, R))} .
$$


for any $f \in L^{p}(B(\bar{x}, R))$. The same argument in the proof of Theorem 29 also gives

$$
\begin{aligned}
\left\|C_{\nu, a} f\right\|_{L^{p}(B(\bar{x}, r))} & \leq c \eta_{a, \Omega_{n+2}, \Omega_{n+3}}^{*}\left(c_{1} r\right)\|f\|_{L^{q}(B(\bar{x}, r))} \\
& \leq \varepsilon\|f\|_{L^{q}(B(\bar{x}, r))}
\end{aligned}
$$

for $a \in V M O_{l o c}\left(\Omega_{n+2}, \Omega_{n+3}\right)$, and $r$ small enough so that $c \eta_{a, \Omega_{n+2}, \Omega_{n+3}}^{*}\left(c_{1} r\right)<$ $\varepsilon$, so we are done.

Theorem 31 (Positive commutators of nonsingular integrals) Let $K, \widetilde{K}$ be as in Assumption (H7), with $K$ satisfying condition (49) with $\nu=0$. Assume that the operator

$$
T f(x)=\int_{B(\bar{x}, R)} \widetilde{K}(x, y) f(y) d \mu(y)
$$

is continuous on $L^{p}(B(\bar{x}, R))$ for any $p \in(1, \infty)$. For $a \in B M O_{l o c}\left(\Omega_{n+2}, \Omega_{n+3}\right)$, set

$$
C_{a} f(x)=\int_{B(\bar{x}, R)} \widetilde{K}(x, y)|a(x)-a(y)| f(y) d \mu(y),
$$

then

$$
\left\|C_{a} f\right\|_{L^{p}(B(\bar{x}, R))} \leq c\|a\|_{B M O_{l o c}\left(\Omega_{n+2}, \Omega_{n+3}\right)}\|f\|_{L^{p}(B(\bar{x}, R))}
$$

for any $f \in L^{p}(B(\bar{x}, R)), p \in(1, \infty)$.

Moreover, if $a \in V M O_{l o c}\left(\Omega_{n+2}, \Omega_{n+3}\right)$ for any $\varepsilon>0$ there exists $r>0$ such that for any $f \in L^{p}(B(\bar{x}, r))$ we have

$$
\left\|C_{a} f\right\|_{L^{p}(B(\bar{x}, r))} \leq \varepsilon\|f\|_{L^{p}(B(\bar{x}, r))} .
$$

The constant $c$ depends on $n$, the constants involved in the assumptions on $K$, and the $L^{p}-L^{p}$ norm of the operator $T$ (but not explicitly on $R$ ); the constant $r$ also depends on the $V M O_{l o c}\left(\Omega_{n+2}, \Omega_{n+3}\right)$ modulus of $a$.

Proof. The proof is very similar to that of Theorem 30. Here we need to apply Theorem 47 which holds in spaces of homogeneous type.

Remark 32 The presence of an absolute value inside the integral in (61) and (62) make the corresponding commutator theorems more flexible than the analogue for singular integrals. Namely, if Theorem 30 or 31 applies to a kernel K, it also applies to any other positive kernel equivalent to $K$, differently from what happens for singular integrals, for which the cancellation property is crucial.

\section{Local maximal operators}

In this section we briefly deal with the local maximal operator in locally homogeneous spaces. The result is substantially already known (see for instance 24]), but for the sake of completeness we state it explicitly with the language and notation of this paper. 
Definition 33 Fix $\Omega_{n}, \Omega_{n+1}$ and, for any $f \in L^{1}\left(\Omega_{n+1}\right)$ define the local maximal function

$$
M_{\Omega_{n}, \Omega_{n+1}} f(x)=\sup _{r \leq r_{n}} \frac{1}{\mu(B(x, r))} \int_{B(x, r)}|f(y)| d \mu(y) \text { for } x \in \Omega_{n}
$$

where $r_{n}=2 \varepsilon_{n} /\left(2 B_{n}+3 B_{n}^{2}\right)$, with $B_{n}$ as in (7).

The following Vitali covering lemma holds, with the usual proof (see e.g. [19, Chap. 3]), thanks to the fact that by our restriction on $x$ and $r$ we can apply the local doubling condition to the involved balls:

Lemma 34 Let $E$ be a measurable subset of $\Omega_{n}$ that is covered by the union of a family of balls $B\left(x_{\alpha}, r_{\alpha}\right)$ centered at points of $\Omega_{n}$ and with radii $r_{\alpha} \leq r_{n}$. Then one can select a disjoint countable subcollection $\left\{B\left(x_{\alpha_{j}}, r_{\alpha_{j}}\right)\right\}_{j=1}^{\infty}$ so that

$$
E \subset \bigcup_{j=1}^{\infty} B\left(x_{\alpha_{j}}, K r_{\alpha_{j}}\right) \text { with } K=\left(2 B_{n}+3 B_{n}^{2}\right) \text {, }
$$

and, for some constant $c$ depending on $n$,

$$
\sum_{j=1}^{\infty} \mu\left(B\left(x_{\alpha_{j}}, r_{\alpha_{j}}\right)\right) \geq c \mu(E)
$$

Then, again repeating the standard proof, one can establish the following:

Theorem 35 Let $f$ be a measurable function defined on $\Omega_{n+1}$. The following hold:

(a) If $f \in L^{p}\left(\Omega_{n+1}\right)$ for some $p \in[1, \infty]$, then $M_{\Omega_{n}, \Omega_{n+1}} f$ is finite almost everywhere in $\Omega_{n}$;

(b) if $f \in L^{1}\left(\Omega_{n+1}\right)$, then for every $t>0$,

$$
\mu\left(\left\{x \in \Omega_{n}:\left(M_{\Omega_{n}, \Omega_{n+1}} f\right)(x)>t\right\}\right) \leq \frac{c_{n}}{t} \int_{\Omega_{n+1}}|f(y)| d \mu(y) ;
$$

(c) if $f \in L^{p}\left(\Omega_{n+1}\right), 1<p \leq \infty$, then $M_{\Omega_{n}, \Omega_{n+1}} f \in L^{p}\left(\Omega_{n}\right)$ and

$$
\left\|M_{\Omega_{n}, \Omega_{n+1}} f\right\|_{L^{p}\left(\Omega_{n}\right)} \leq c_{n, p}\|f\|_{L^{p}\left(\Omega_{n+1}\right)} .
$$

\section{Quasisymmetric quasidistances}

In this section we want to extend the main results of the previous theory to the more general case of a quasisymmetric $\rho$. We stress the fact that the results we are going to extend are those of Sections $[5$ to 8 but not the construction of dyadic cubes of Section 3 . 
Definition 36 (Quasisymmetric locally homogeneous space) We make the following assumptions.

(K1) Let $\Omega$ be a set, endowed with a function $\rho: \Omega \times \Omega \rightarrow[0, \infty)$ such that for any $x, y \in \Omega \rho(x, y)=0 \Leftrightarrow x=y$.

For any $x \in \Omega, r>0$, let us define the ball

$$
B(x, r)=\{y \in \Omega: \rho(x, y)<r\}
$$

and the coball

$$
B^{\prime}(x, r)=\{y \in \Omega: \rho(y, x)<r\} .
$$

Let us define a topology in $\Omega$ saying that $A \subset \Omega$ is open if for any $x \in A$ there exists $r>0$ such that $B(x, r) \subset A$. Also, we will say that $E \subset \Omega$ is bounded if $E$ is contained in some ball. Let us assume that:

(K2') $\rho(x, y)$ is a continuous function of $x$ for any fixed $y \in \Omega$ and a continuous function of $y$ for any fixed $x \in \Omega$.

(H3) Let $\mu$ be a positive regular Borel measure in $\Omega$.

(K4) Assume there exists an increasing sequence $\left\{\Omega_{n}\right\}_{n=1}^{\infty}$ of bounded measurable subsets of $\Omega$, such that:

$$
\bigcup_{n=1}^{\infty} \Omega_{n}=\Omega
$$

and such for, any $n=1,2,3, \ldots$ :

(i) the closure of $\Omega_{n}$ in $\Omega$ is compact;

(ii) there exists $\varepsilon_{n}>0$ such that

$$
\begin{aligned}
& \left\{x \in \Omega: \rho(x, y)<2 \varepsilon_{n} \text { for some } y \in \Omega_{n}\right\} \subset \Omega_{n+1} \\
& \left\{x \in \Omega: \rho(y, x)<2 \varepsilon_{n} \text { for some } y \in \Omega_{n}\right\} \subset \Omega_{n+1}
\end{aligned}
$$

(K5) there exist $A_{n}, B_{n} \geq 1$ such that for any $x, y, z \in \Omega_{n}$

$$
\begin{aligned}
& \rho(x, y) \leq A_{n} \rho(y, x) ; \\
& \rho(x, y) \leq B_{n}(\rho(x, z)+\rho(z, y)) ;
\end{aligned}
$$

(H6) there exists $C_{n}>1$ such that for any $x \in \Omega_{n}, 0<r \leq \varepsilon_{n}$ we have

$$
0<\mu(B(x, 2 r)) \leq C_{n} \mu(B(x, r))<\infty .
$$

(Note that for $x \in \Omega_{n}$ and $r \leq \varepsilon_{n}$ we also have $\left.B(x, 2 r) \subset \Omega_{n+1}\right)$.

We will say that $\left(\Omega,\left\{\Omega_{n}\right\}_{n=1}^{\infty}, \rho, \mu\right)$ is a quasisymmetric locally homogeneous space if assumptions (K1), (K2'), (H3), (K4), (K5), (H6) hold.

With a proof very similar to that of Proposition 4 in Section 2 we can prove the following: 
Proposition 37 Condition (K2') is equivalent to the validity of both the following

(K2) (a) the balls and coballs are open with respect to this topology;

(K2) (b) for any $x \in \Omega$ and $r>0$ the closure of $B(x, r)$ is contained in $\{y \in \Omega: \rho(x, y) \leq r\}$ and the closure of $B^{\prime}(x, r)$ is contained in $\{y \in \Omega: \rho(y, x) \leq r\}$.

It is also immediate to check the following

Proposition 38 If $\left(\Omega,\left\{\Omega_{n}\right\}_{n=1}^{\infty}, \rho, \mu\right)$ is a quasisymmetric locally homogeneous space and

$$
\rho^{*}(x, y)=\rho(x, y)+\rho(y, x),
$$

then $\left(\Omega,\left\{\Omega_{n}\right\}_{n=1}^{\infty}, \rho^{*}, \mu\right)$ is a locally homogeneous space, and its constants can be bounded in terms of the constants of $\left(\Omega,\left\{\Omega_{n}\right\}_{n=1}^{\infty}, \rho, \mu\right)$.

We now want to apply the results we have proved in Sections 5 to 8 to show that similar results hold in a quasisymmetric locally homogeneous space. Let us discuss in detail one of these results, the others being similar.

Theorem 39 ( $L^{p}$ and $C^{\eta}$ estimates for singular integrals) Theorem 21 still holds if $\left(\Omega,\left\{\Omega_{n}\right\}_{n=1}^{\infty}, \rho, \mu\right)$ is a quasisymmetric locally homogeneous space.

Proof. The key observation is that if the kernel $K$ satisfies conditions (i), (ii), (iii) in Proposition 19 with respect to $\rho$, it also satisfies them with respect to any equivalent function, in particular with respect to $\rho^{*}$; this follows by a standard computation, and implies the validity of $L^{p}$ estimates, by Theorem 21. As to $C^{\eta}$ estimates, let us first note that $\rho$ and $\rho^{*}$ define the same space $C^{\eta}$, with equivalent norms. Moreover, if $K$ satisfies the condition

$$
\widetilde{h}(x) \equiv \lim _{\varepsilon \rightarrow 0} \int_{\rho^{\prime}(x, y)>\varepsilon} \widetilde{K}(x, y) d \mu(y) \in C^{\gamma}\left(\Omega_{n+1}\right)
$$

for some $\gamma>0$ and some $\rho^{\prime}$ equivalent to $\rho$, this $\rho^{\prime}$ is also equivalent to $\rho^{*}$, so $\widetilde{h}$ satisfies the Hölder continuity assumption required by Theorem 21 hence Hölder estimates hold.

A similar argument shows that Theorems 24 and25still holds if $\left(\Omega,\left\{\Omega_{n}\right\}_{n=1}^{\infty}, \rho, \mu\right)$ is a quasisymmetric locally homogeneous space.

To deal with commutators we first need to make the following remark about $B M O$ spaces.

Let us denote by $B_{r}, \widetilde{B}_{r}$ the balls with respect to any two equivalent functions $\rho, \widetilde{\rho}$ satisfying the axioms of quasisymmetric locally doubling spaces. Then for any $x_{0} \in \Omega_{n}, r \leq \varepsilon_{n}$, any $\tau \in \mathbb{R}$, we can write, by the equivalence of $\rho, \widetilde{\rho}$

$$
\frac{1}{\left|B_{r}\left(x_{0}\right)\right|} \int_{B_{r}\left(x_{0}\right)}|u(x)-\tau| d \mu(x) \leq \frac{1}{\left|\widetilde{B}_{c_{1} r}\left(x_{0}\right)\right|} \int_{\widetilde{B}_{c_{2} r}\left(x_{0}\right)}|u(x)-\tau| d \mu(x)
$$

by the local doubling condition

$$
\leq \frac{c_{3}}{\left|\widetilde{B}_{c_{2} r}\left(x_{0}\right)\right|} \int_{\widetilde{B}_{c_{2} r}\left(x_{0}\right)}|u(x)-\tau| d \mu(x) .
$$


Choosing $\tau=\frac{1}{\left|\widetilde{B}_{c_{2} r}\left(x_{0}\right)\right|} \int_{\widetilde{B}_{c_{2} r}\left(x_{0}\right)} u(x) d \mu(x)$ and recalling that for any $\tau$ we have

$\frac{1}{\left|B_{r}\left(x_{0}\right)\right|} \int_{B_{r}\left(x_{0}\right)}\left|u(x)-u_{B_{r}\left(x_{0}\right)}\right| d \mu(x) \leq 2 \cdot \frac{1}{\left|B_{r}\left(x_{0}\right)\right|} \int_{B_{r}\left(x_{0}\right)}|u(x)-\tau| d \mu(x)$

we get the equivalence between the norms $\|u\|_{B M O_{l o c}\left(\Omega_{n}, \Omega_{n+1}\right)}$ with respect to $\rho$ and $\widetilde{\rho}$, and an analogous equivalence between $V M O_{l o c}$ moduli. Applying this argument to the quasisymmetric function $\rho$ and its symmetrized $\rho^{*}$ we immediately get that also the commutator theorems 29, 30, 31 still hold if $\left(\Omega,\left\{\Omega_{n}\right\}_{n=1}^{\infty}, \rho, \mu\right)$ is a quasisymmetric locally homogeneous space.

Finally, the extension of Theorem 35 to the setting of quasisymmetric locally homogeneous spaces is immediate, since the maximal functions defined with respect to equivalent quasisymmetric quasidistances are equivalent, hence the result in the symmetric case implies that for the quasisymmetric case.

\section{Appendix. Known results for spaces of ho- mogeneous type}

In this Appendix we collect all the results about spaces of homogeneous type which we have applied throughout the paper. We first recall the basic

Definition 40 Let $X$ be a set endowed with a function $\rho: X \times X \rightarrow[0, \infty)$ such that for some constant $B \geq 1$, any $x, y, z \in X$ :

$$
\begin{aligned}
& \rho(x, y)=0 \Longleftrightarrow x=y ; \\
& \rho(x, y)=\rho(y, x) ; \\
& \rho(x, y) \leq B(\rho(x, z)+\rho(z, y)) .
\end{aligned}
$$

Assume that the $\rho$-balls are open with respect to the topology they induce. Let $\mu$ be a positive Borel measure on $X$, satisfying the doubling condition

$$
0<\mu(B(x, 2 r)) \leq C \mu(B(x, r))<\infty
$$

for any $x \in X, r>0$. Then we say that $(X, \rho, \mu)$ is a space of homogeneous type.

Dependence of the constants. We will say that some constant depends on $X$ to say that it depends on the constants $B, C$.

\section{1 $L^{p}$ and $C^{\alpha}$ estimates for singular integrals on spaces of homogeneous type}

Theorem 41 ( $L^{p}$ continuity of singular integrals) Let $(X, \rho, \mu)$ be a homogeneous space, $\mu$ a regular measure. Let $K: X \times X \backslash\{x=y\} \rightarrow \mathbb{R}$ a kernel satisfying the following conditions:

the standard estimates (48) with $\nu=0$, for any $x, y \in X$, and (49), for any $x_{0}, x, y \in X$, with $\rho\left(x_{0}, y\right) \geq M \rho\left(x_{0}, x\right), M>1, \nu=0, \beta>0$; 
the cancellation property

$$
\left|\int_{r \leq \rho^{\prime}(x, y) \leq R} K(y, x) d \mu(y)\right|+\left|\int_{r \leq \rho^{\prime}(x, y) \leq R} K(x, y) d \mu(y)\right| \leq C
$$

for any $R>r>0, x \in X$, where $\rho^{\prime}$ is any quasidistance equivalent to $\rho$. Let $T_{\varepsilon}$ be the truncated operator defined as

$$
T_{\varepsilon} f=\int_{\rho^{\prime}(x, y) \geq \varepsilon} K(x, y) f(y) d \mu(y)
$$

for any $f \in C_{0}^{\eta}(X)$ (with $\eta$ small enough so that $C_{0}^{\eta}(X)$ is dense in $L^{p}$ for $p \in[1, \infty)$ ). Then $T_{\varepsilon}$ can be extended to a linear continuous operator from $L^{p}$ into $L^{p}$ for every $p \in(1, \infty)$, and

$$
\left\|T_{\varepsilon} f\right\|_{L^{p}} \leq c\|f\|_{L^{p}}
$$

where the constant $c$ depends on $X, p$ and all the constants involved in the assumptions, but not on $\varepsilon$. Moreover, if for a.e. $x \in X$ there exists the limit

$$
\lim _{\varepsilon \rightarrow 0} \int_{\rho^{\prime}(x, y) \geq \varepsilon} K(x, y) d \mu(y),
$$

then the above $L^{p}$ estimate holds also for the operator

$$
T f(x)=\lim _{\varepsilon \rightarrow 0} \int_{\rho^{\prime}(x, y) \geq \varepsilon} K(x, y) f(y) d \mu(y) .
$$

Finally, the operator $T$ satisfies a weak $(1,1)$-estimate:

$$
\mu(\{x \in X:|T f(x)|>t\}) \leq \frac{c}{t}\|f\|_{L^{1}(X)} .
$$

The above result follows, for instance, from the results contained in [16] and [19]; see also [8, Thm. 4.1, Thm. 4.5] where this theorem is explicitly derived from the aforementioned results.

Theorem 42 ( $C^{\alpha}$ continuity of singular integrals) (See [5, Thm. 2.7]). Let $(X, \rho, \mu)$ be a bounded homogeneous space, and let $K: X \times X \backslash\{x=y\} \rightarrow \mathbb{R}$ a kernel satisfying the following conditions:

the standard estimate (48) with $\nu=0$, any $x, y \in X$, and (49) with $\nu=0$, for any $x_{0}, x, y \in X$, with $\rho\left(x_{0}, y\right) \geq M \rho\left(x_{0}, x\right), M>1, \beta>0$;

the cancellation property: for any $r>0$

$$
\left|\int_{\rho^{\prime}(x, y) \geq r} K(y, x) d \mu(y)\right|+\left|\int_{\rho^{\prime}(x, y) \geq r} K(x, y) d \mu(y)\right| \leq C,
$$

where $\rho^{\prime}$ is any quasidistance on $X$, equivalent to $\rho$. Assume that for every $f \in C^{\alpha}(X)$ and $x \in X$ the following limit exists:

$$
T f(x)=\lim _{\varepsilon \rightarrow 0} T_{\varepsilon} f(x)=\lim _{\varepsilon \rightarrow 0} \int_{\rho^{\prime}(x, y) \geq \varepsilon} K(x, y) f(y) d y
$$


and $T(1)(x) \in C^{\eta}(X)$, for some $\eta \in(0,1]$ Then the operator $T$ is continuous on $C^{\alpha}(X)$; more precisely:

$$
|T f|_{C^{\alpha}(X)} \leq c\|f\|_{C^{\alpha}(X)} \text { for every } \alpha<\beta, \alpha \leq \eta
$$

for some constant $c$ depending on $X, \alpha$, and all the constants involved in the assumptions (recall $\beta$ is the exponent appearing in assumption (49)). Moreover,

$$
\|T f\|_{L^{\infty}} \leq c\|f\|_{C^{\alpha}(X)},
$$

where $c$ also depending on diamX.

\section{2 $L^{p}$ and $C^{\alpha}$ estimates for fractional integrals on spaces of homogeneous type}

Theorem 43 ( $L^{p}$ estimate for fractional integrals) Let $(X, \rho, \mu)$ be a space of homogeneous type, and for $\alpha \in(0,1)$, let

$$
\begin{gathered}
0 \leq K_{\alpha}(x, y) \leq \frac{C}{\mu(B(x, \rho(x, y)))^{1-\alpha}} \text { for } x \neq y, K_{\alpha}(x, x)=0 \\
I_{\alpha} f(x)=\int_{X} K_{\alpha}(x, y) f(y) d \mu(y)
\end{gathered}
$$

for any measurable $f: X \rightarrow \mathbb{R}$ for which the integral makes sense. Then, for any $p \in\left(1, \frac{1}{\alpha}\right), \frac{1}{q}=\frac{1}{p}-\alpha$ there exists a constant depending on $X, \alpha, p$ and the constant $C$ in the assumptions on $K_{\alpha}$, such that

$$
\left\|I_{\alpha} f\right\|_{L^{q}(X)} \leq c\|f\|_{L^{p}(X)}
$$

for any $f \in L^{p}(X)$.

The above result is due to Gatto-Vagi, see [22], 23].

Theorem 44 ( $C^{\alpha}$ estimate for fractional integrals) (See [5, Thm 2.11]). Let $(X, \rho, \mu)$ be a bounded space of homogeneous type, and let $K(x, y)$ be a kernel satisfying for some $\nu \in(0,1)$ the standard estimates 48$)$ for any $x, y \in X$, and (49), for any $x_{0}, x, y \in X$, with $\rho\left(x_{0}, y\right) \geq M \rho\left(x_{0}, x\right), M>1, \beta>0$; let

$$
I_{\nu} f(x)=\int_{X} K_{\nu}(x, y) f(y) d \mu(y) .
$$

Then, for any $\alpha<\min (\beta, \nu)$ we have

$$
\left\|I_{\nu} f\right\|_{C^{\alpha}(X)} \leq c\|f\|_{C^{\alpha}(X)} .
$$

The constant $c$ depends on $X, \alpha, \operatorname{diam} X$, and the constants involved in the assumptions on $K$.

The above two results about fractional integrals are proved in the quoted papers under some additional assumptions on the space (e.g., the absence of "atoms", that is points of positive measure); however, these statements can be easily proved in full generality. 


\subsection{Commutator theorems}

The original commutator theorem we are interested in is the one proved by Coifman-Rochberg-Weiss [18 for classical Calderón-Zygmund operators. The extension of this result to spaces of homogeneous type, both bounded and unbounded, has been proved in [8, Thm 2.5, Thm. 3.1]:

Theorem 45 (Commutators of singular integrals) Let $(X, \rho, \mu)$ be a homogeneous space and let all the assumptions of Theorem 41 be in force. Let

$$
T f(x)=\lim _{\varepsilon \rightarrow 0} \int_{X, \rho^{\prime}(x, y)>\varepsilon} K(x, y) f(y) d \mu(y)
$$

and, for $a \in B M O(X)$ let

$$
C_{a} f(x)=T(a f)(x)-a(x) T f(x) .
$$

Then, for any $p \in(1, \infty)$

$$
\left\|C_{a} f\right\|_{L^{p}(X)} \leq c\|a\|_{B M O(X)}\|f\|_{L^{p}(X)}
$$

for some constant $c$ depending on $X, p$, and the constants involved in the assumptions on $K$, but not on $f, a$.

Next, let us recall the analog results for fractional or more general type of nonsingular integral operators. A key point in the following result is the presence of an absolute value inside the integral, which is allowed by the positivity of the kernel:

Theorem 46 (Commutators of fractional integrals) Let $(X, \rho, \mu)$ be a homogeneous space and let $K_{\alpha}, I_{\alpha}$ be as in Theorem 43. For any function $a \in$ $B M O(X)$, let

$$
C_{a} f(x)=\int_{X} K_{\alpha}(x, y)|a(x)-a(y)| f(y) d \mu(y)
$$

be the "positive commutator" of $I_{\alpha}$ with $a$. Then for any $p \in\left(1, \frac{1}{\alpha}\right), \frac{1}{q}=\frac{1}{p}-\alpha$ there exists $c=c(X, \alpha, p)$ such that

$$
\left\|C_{a} f\right\|_{L^{q}(X)} \leq c\|a\|_{B M O(X)}\|f\|_{L^{p}(X)} .
$$

The above theorem has been first proved in [9, Thm. 2.11] under an extra assumption on the space $(X, \rho)$ and then, in this full generality, in [1, Thm. 3.3, Thm. 3.7].

An analog result holds for any abstract operator with positive kernel, which we already know to be $L^{p}$ continuous: 
Theorem 47 (See [1, Thm. 0.1]). Let $(X, \rho, \mu)$ be a homogeneous space and $K(x, y)$ be a nonnegative kernel such that the operator

$$
T f(x)=\int_{X \backslash\{x\}} K(x, y) f(y) d \mu(y)
$$

maps $L^{p}(X)$ into $L^{p}(X)$ for $p \in(1, \infty)$. Also, assume $K$ satisfies the standard estimate (49) for $\nu=0$, some $\beta>0, M>1$, any $x_{0}, x, y \in X$ with $\rho\left(x_{0}, y\right)>$ $M \rho\left(x_{0}, x\right)$. For $a \in B M O(X)$, let

$$
C_{a} f(x)=\int_{X} K(x, y)|a(x)-a(y)| f(y) d \mu(y) .
$$

Then there exists $c=c(X, p)$ such that

$$
\left\|C_{a} f\right\|_{L^{p}(X)} \leq c\|a\|_{B M O(X)}\|f\|_{L^{p}(X)} .
$$

\section{References}

[1] M. Bramanti: Commutators of integral operators with positive kernels. Le Matematiche (Catania) 49 (1994), no. 1, 149-168 (1995).

[2] M. Bramanti: Singular integrals in nonhomogeneous spaces: $L^{2}$ and $L^{p}$ continuity from Hölder estimates. Rev. Mat. Iberoam. 26 (2010), no. 1, $347-366$.

[3] M. Bramanti, L. Brandolini: $L^{p}$-estimates for nonvariational hypoelliptic operators with VMO coefficients. Trans. Amer. Math. Soc. 352 (2000), no. $2,781-822$.

[4] M. Bramanti-L. Brandolini: Estimates of BMO type for singular integrals on spaces of homogeneous type and applications to hypoelliptic PDES. Revista Matematica Iberoamericana, 21 (2005), no. 2, 511-556.

[5] M. Bramanti, L. Brandolini: Schauder estimates for parabolic nondivergence operators of Hörmander type. J. Differential Equations 234 (2007), no. $1,177-245$.

[6] M. Bramanti, L. Brandolini, M. Pedroni: Basic properties of nonsmooth Hörmander's vector fields and Poincaré's inequality. Submitted preprint, 2008. arXiv:0809.2872

[7] M. Bramanti, L. Brandolini, M. Pedroni: On the lifting and approximation theorem for nonsmooth vector fields. ArXiv: 1002.1331v1 5 feb 2010. To appear on Indiana University Mathematics Journal.

[8] M. Bramanti, M. C. Cerutti: Commutators of singular integrals on homogeneous spaces. Boll. Un. Mat. Ital. B (7) 10 (1996), no. 4, 843-883. 
[9] M. Bramanti, M. C. Cerutti: Commutators of singular integrals and fractional integrals on homogeneous spaces. Harmonic analysis and operator theory (Caracas, 1994), 81-94, Contemp. Math., 189, Amer. Math. Soc., Providence, RI, 1995.

[10] M. Bramanti, G. Cupini, E. Lanconelli, E. Priola: Global $L^{p}$ estimates for degenerate Ornstein-Uhlenbeck operators. Mathematische Zeitschrift, 266, n. 4 (2010), pp. 789-816.

[11] M. Bramanti, M. Zhu: $L^{p}$ and $C^{\alpha}$ estimates for nonvariational operators structured on Hörmander's vector fields with drift. Preprint.

[12] A. Carbonaro, G. Mauceri, S. Meda, $H^{1}$ and BMO for certain locally doubling metric measure spaces. Ann. Sc. Norm. Super. Pisa Cl. Sci. (5) 8 (2009), no. 3, 543-582.; arXiv:0808.0146

[13] R. R. Coifman, R. Rochberg, G. Weiss: Factorization theorems for Hardy spaces in several variables. Ann. of Math. (2) 103 (1976), no. 3, 611-635.

[14] F. Chiarenza, M. Frasca, P. Longo: Interior $W^{2, p}$-estimates for nondivergence elliptic equations with discontinuous coefficients. Ricerche Mat. 40 (1991), no. 1, 149-168.

[15] F. Chiarenza, M. Frasca, P. Longo: $W^{2, p}$-solvability of the Dirichlet problem for nondivergence elliptic equations with VMO coefficients. Trans. Amer. Math. Soc. 336 (1993), no. 2, 841-853.

[16] M. Christ: A $T(b)$ theorem with remarks on analytic capacity and the Cauchy integral. Colloq. Math. 60/61 (1990), no. 2, 601-628.

[17] M. Christ: Lectures on singular integral operators, CBMS Regional Conf. Ser. in Math., 77 (1990).

[18] R. R. Coifman, R. Rochberg, G. Weiss: Factorization theorems for Hardy spaces in several variables. Ann. of Math. (2) 103 (1976), no. 3, 611-635.

[19] R. R. Coifman, G. Weiss: Analyse harmonique non-commutative sur certains espaces homogènes. Lecture Notes in Mathematics, Vol. 242. SpringerVerlag, Berlin-New York, 1971.

[20] H. Federer. Geometric measure theory. Die Grundlehren der mathematischen Wissenschaften, Band 153 Springer-Verlag New York Inc., New York 1969 .

[21] B. Franchi, E. Lanconelli: Hölder regularity theorem for a class of linear nonuniformly elliptic operators with measurable coefficients. Ann. Scuola Norm. Sup. Pisa Cl. Sci. (4) 10 (1983), no. 4, 523-541.

[22] E. Gatto, S. Vági: Fractional integrals on spaces of homogeneous type. Analysis and partial differential equations, 171-216, Lecture Notes in Pure and Appl. Math., 122, Dekker, New York, 1990. 
[23] E. Gatto, S. Vági: On molecules and fractional integrals on spaces of homogeneous type with finite measure. Studia Math. 103 (1992), no. 1, 25-39.

[24] M. Kronz: Some function spaces on spaces of homogeneous type. Manuscripta Math. 106 (2001), no. 2, 219-248.

[25] R. A. Macías, C. Segovia: Lipschitz functions on spaces of homogeneous type. Adv. in Math. 33 (1979), no. 3, 257-270.

[26] A. Nagel, E.M. Stein, S. Wainger: Balls and metrics defined by vector fields. I. Basic properties. Acta Math. 155 (1985), no. 1-2, 103-147.

[27] L.P. Rothschild, E.M. Stein: Hypoelliptic differential operators and nilpotent groups. Acta Math. 137 (1976), no. 3-4, 247-320.

[28] X. Tolsa: $B M O, H^{1}$, and Calderón-Zygmund operators for non doubling measures. Math. Ann. 319 (2001), no. 1, 89-149.

[29] A. Volberg: Calderón-Zygmund capacities and operators on nonhomogeneous spaces. CBMS Regional Conference Series in Mathematics, 100. Am. Math. Soc., Providence, RI, 2003.

Marco Bramanti

Dipartimento di Matematica, Politecnico di Milano

Via Bonardi 9. 20133 Milano. ITALY

marco.bramanti@polimi.it

Maochun Zhu

Department of Applied Mathematics, Northwestern Polytechincal University 127 West Youyi Road. 710072 Xi'an. P. R. CHINA

zhumaochun2006@126.com 